\title{
Effects of strength training with variable elastic resistance across the lifespan: a systematic review
}

\author{
Efectos del entrenamiento de la fuerza con resistencia variable \\ elástica a lo largo de la vida: una revisión sistemática
Juan C. Colado ${ }^{1,2}$, Ranulfo Mena ${ }^{1}$, Joaquín Calatayud ${ }^{1}$, Pedro Gargallo ${ }^{2}$, Jorge Flández ${ }^{3}$ Phil Page $^{4}$
1 Research Unit in Sport and Health, University of Valencia (Spain).
2 Research Group in Prevention and Health in Exercise and Sport, University of Valencia (Spain).
3 Institute of Education Sciences. Physical Education and Sport Degree, Austral University of Chile (Valdivia, Chile). \\ 4 Franciscan Missionaries of Our Lady University, Baton Rouge, Louisiana (USA). \\ CORRESPONDENCIA: \\ Juan C. Colado \\ juan.colado@uv.es \\ Recepción: julio 2018 • Aceptación: enero 2020

\section{CÓMO CITAR EL ARTÍCULO:} \\ Colado, J. C., Mena, R., Calatayud, J., Gargallo, P., Flández, J., \& \\ Page, P. (2020). Effects of strength training with variable elastic \\ resistance across the lifespan: a systematic review. Cultura, Cien- \\ cia y Deporte, 15(44), 147-164.
}

\begin{abstract}
The benefits of strength training programs with isotonic free weights or machines have been well-documented in all age groups. However, exercise and healthcare professionals sometime question whether it is possible to obtain the same results with devices of variable resistance, such as elastic bands. To answer this question, the purpose of this systematic review was to identify and summarize the positive effects of elastic resistance exercises used across the lifespan on health outcomes including body composition, functional and performance capacity, and biochemical variables. A secondary aim was to identify common dosage parameters of strength training programs using elastic resistance.
\end{abstract}

Key words: elastic bands; functional capacity, body composition; health biomarkers.

\section{Resumen}

Los beneficios de los programas de entrenamiento de la fuerza con peso libre y máquinas isocinéticas del tipo isotónico han sido bien documentados en todas las franjas de edad. Sin embargo, los profesionales del ejercicio y la salud algunas veces se preguntan si es posible obtener los mismos resultados con dispositivos de resistencia variable, como por ejemplo las bandas elásticas. Para responder a esta pregunta, el objetivo de esta revisión sistemática fue identificar y resumir los efectos positivos de los ejercicios de fuerza con elásticos empleados a lo largo de las diferentes etapas de la vida sobre resultados relacionados con la salud, incluyendo la composición corporal, capacidad funcional, rendimiento físico y algunas variables bioquímicas. Un objetivo secundario fue identificar los parámetros de dosificación comunes de los programas de entrenamiento de la fuerza usando resistencia elástica.

Palabras clave: bandas elásticas; capacidad funcional; composición corporal; biomarcadores de salud. 


\section{Introduction}

Resistance training has been recommended across the lifespan for muscular fitness in support of health-realted physical fitness. Exercise and health professionals need effective resistance training devices that stimulate these positive adaptations while promoting adherence to exercise (Capodaglio, Ferri \& Scaglioni, 2005; Capodaglio et al., 2002; Gómez-Álvarez, JofréHermosilla, Matus-Castillo, \& Pavez-Adasme, 2019). Many strength training devices are available, each with its own advantages and disadvantages.

As the most common and traditional devices for external reistance in training programs, free weights and machines have been shown beneficial for physical function, body composition and other health-related variables (Kwak, Kim \& Lee, 2016; Liao, Chung \& Chen, 2017; Winters-Stone \& Snow, 2006). However, free weights and machines often require special facilities and/or great cost, thus limiting allowing access for everyone (Colado \& Triplett, 2008). Furthermore, some individuals may fear using free weights and machines because these are commonly associated with high physical demands (Jakobsen, Sundstrup, Andersen, Aagaard \& Andersen, 2013) or possible injury.

In contrast, variable resistance training with elastic bands provides a more user-friendly, portable and less-expensive alternative to traditional isotonic resistance training. Furthermore, evidence suggests that elastic resistance can improve muscular hypertrophy, strength and power (Suchomel, Nimphius, Bellon \& Stone, 2018). Although these elastic devices have traditionally been used for rehabilitation purposes (Page \& Ellenbecker, 2003), the benefits of elastic resistance in apparent healthy populations has been studied as well (Colado et al., 2010). Researchers have demonstrated that variable elastic resistance can provide similar muscle activation as constant-resistance equivalents when matched in intensity, as well as provide a mechanical advantage over the "sticking point" of free weights and machines. (Aboodarda, Hamid, Che Muhamed, Ibrahim \& Thompson, 2013; Aboodarda, Page \& Behm, 2016; Calatayud et al., 2015; Hughes \& Mcbridge, 2005; Kompf \& Arandjelovic, 2016; Matheson, Kernozek, Fater \& Davies, 2001; Soria-Gila, Chirosa, Bautista, Baena \& Chirosa, 2015). In addition, the authors of recent review articles have concluded that elastic resistance training can provide both strength and functional improvements among adults (de Oliveira et al., 2016) and elderly participants (Martins et al., 2013), suggesting elastic resistance may provide benefits across a variety of age groups. However, there are no systematic reviews on the effects or parameters of elastic resistance training throughout the lifespan. For example, while it's been shown that resistance training produces positive results in youths (Granacher et al., 2016), there is not systematic review of elastic resistance training showing benefits in this specific population.

This knowledge would be necessary to provide effective and safe exercise prescriptions for a wider range of individuals. While individual studies have described adaptations of important physiological variables after elastic resistance training (such as biomarkers or body composition), a systematic review of these adaptations awould be beneficial. (Colado, Triplett, Tella, Saucedo \& Abellán, 2009; Colado \& Triplett, 2008; Flandez et al., 2017; Gargallo et al., 2018; Thiebaud et al., 2013).

Therefore, the aim of this systematic review was to identify and summarize the health outcomes of elastic resistance training across the lifespan on body composition, functional capacity and some biochemical variables. A secondary aim was to identify elastic resistance exercise parameters in order to establish safe and effective exercise prescriptions.

\section{Method}

This systematic review is reported using the Preferred Reporting Items for Systematic Reviews and Meta-Analyses (PRISMA) recommendations (Moher, Liberati, Tetzlaff \& Altman, 2009).

\section{Literature search}

We reviewed scientific literature specialized using 9 databases (Web of Science, Pubmed, Sportdiscus, Scopus, Medline, Scielo, Central, Embase and PEDro). The search was limited to full-text, clinical trials studies published in English between 1900 and March 2017. With the search strategy for relevant articles included the following search terms with the Boolean operator "OR": "elastic band", "resistance elastic" , "elastic resistance training" , "elastic tubing", "elastic tubing exercise", "elastic band" , "elastic band exercise" , "exercise band" , "surgical tubing” , "theraband" , "rubber band" , and "elastic straps". In the Boolean operators, "and" was also added to provide the corresponding terminology for each age group analyzed in this study: (i) For children: "kids" or "children" or "child" or "lad" or "baby" or "infant" or "laddie" or "cully" or "chap" or "kiddy" or "nipper" or "boyhood" or "girlhood"; (ii) For young people: "adolescent" or "shaver" or "teenager" or "young people" or "teen" or "junior" or "young"; (iii) For adults: "adults" or "middle age"; (iv) and finally for seniors: "old 


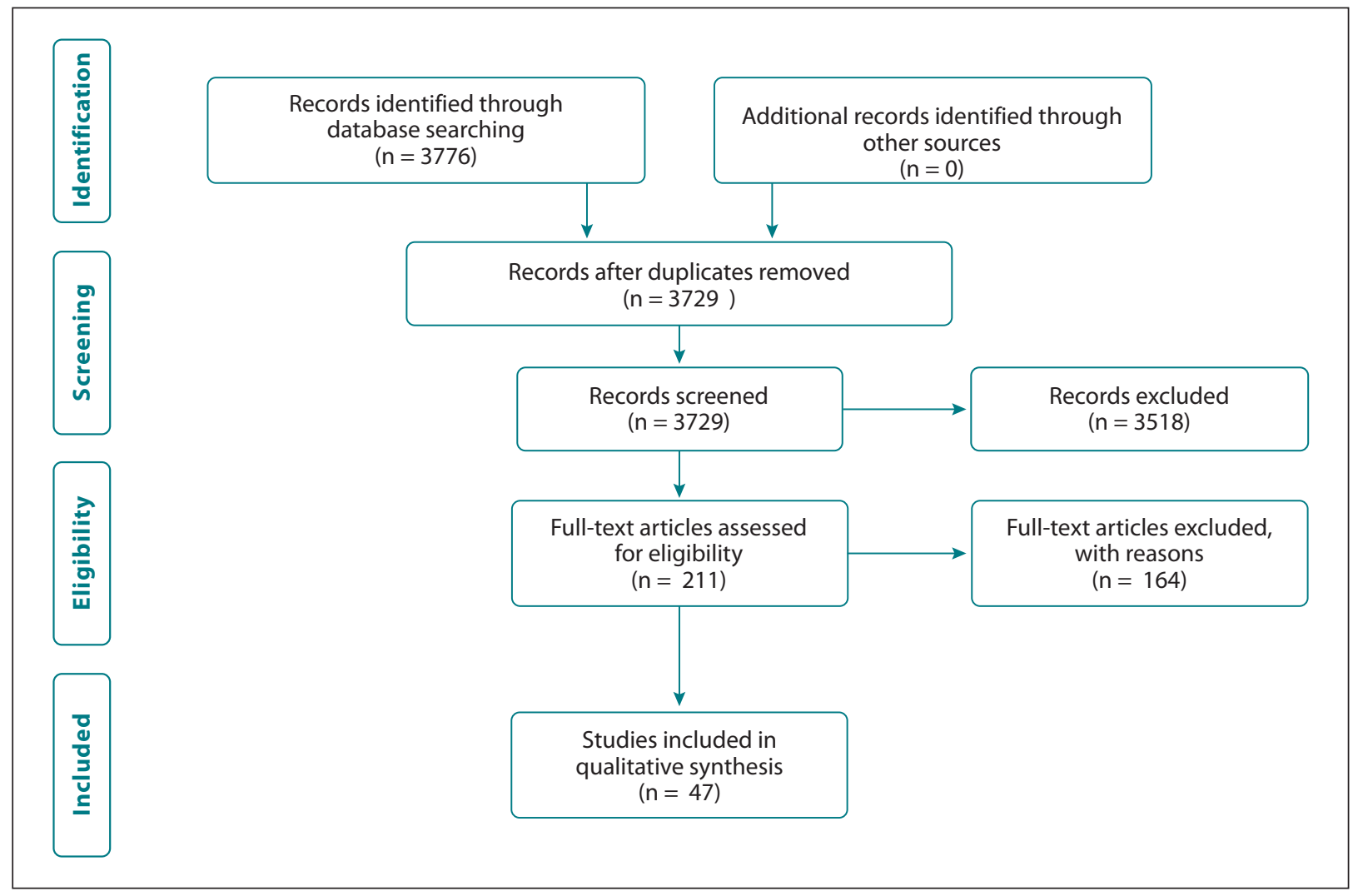

Figure 1. Flow diagram showing the phases of the search and study selection.

man" or "old-timer" or "old person" or or "elderly" or "elder" or "ancient" or "aged" or "older adults" or "aging" or "maturation" or "older" or "old people".

\section{Selection criteria}

The studies had to meet the following inclusion criteria: 1) Healthy subjects; 2) Results in at least one of the following variables: Strength, functional capacity, body composition or biomarkers of health; 3) Results of chronic nature. Exclusion criteria were: 1) Descriptive studies, or validation; 2): Results of acute nature; 3) Subjects with pathology; 4) Studies that have not focused on the variables mentioned above; 5) Studies with highly trained subjects.

\section{Classification of the studies}

The included articles meeting selection criteria were classified with the following data in Table 1: (1) Author and year of publication; (2) Number, Gender, Age, distribution and characteristics of the sample; (3) Duration of the session and the intervention, weekly sessions, number of series, repetitions, rest, intensity and progression; (4) Groups of training and exercises; (5) Variables analyzed: muscle strength, body composi- tion, functional capacity, and biomarkers of health; (6) Results between pre- and post- intervention.

\section{Methodological quality assessment}

The methodological quality of studies, was analyzed using the "PEDro scale", which has been validated by Maher, Sherrington, Herbert, Moseley \& Elkins. (2003). Ten of 11 PEDro criteria were scored "yes (1)" or "no (0)" with a maximum score of 10 (the first criterion of the PEDro scale is not used to calculate the PEDro score). Studies with a score of $\geq 6$ were considere of high methodological quality while a score of $\leq 5$ points was considered to be low quality.

\section{Results}

Figure 1 shows the PRISMA flow chart diagram from the systematic search; 47 studies were included in the analysis. Included studies were classified into 4 age groups: 3 studies in children and adolescents (youths) ( $\leq 18$ years), 6 in young adults (19 and 35 years), 6 in middle-aged adults (45-64 years), and finally, 32 in older adults (>64 years). Table 1 presents the main characteristics of these trials. 


\section{Characteristics of the studies in youths}

Only 3 studies examined elastic resistance training in subjects less than 18 years old (Coskun \& Sahin, 2014; Lubans, Aguiar \& Callister, 2010; Sahin, Aslan \& Demir, 2016). These studies used 2 weekly sessions and included a total of 159 subjects: 65 used elastic bands, 37 free weights and 22 used their own body weight. Two studies used 6 weeks of intervention (Coskun \& Sahin, 2014; Sahin, Aslan \& Demir, 2016), while Lubans, Aguiar \& Callister (2010) used 8 weeks. Coskun \& Sahin (2014) and Sahin, Aslan \& Demir (2016) used a 10 repetitionmaximum (RM) intensity without publishing the time of rest, while Lubans et al. (2010) used between 15 and 18 in the Borg's scale, performing 2 sets of 10 to 12 repetitions with a rest between sets of 60-90 seconds. The duration of the sessions ranged between 30 and 50 minutes. Sahin, Aslan \& Demir (2016) evaluated the effect of using elastic resitance in the squat and the jump, while Coskun \& Sahin (2014) and Lubans, Aguiar \& Callister (2010) used overall body exercises.

\section{Characteristics of the studies in young adults}

Seven studies were included. 136 subjects were studied with adults between 19 to 35 years old: 83 used elastic bands, 12 other devices, and 41 as a control group. The interventions lasted from 6 to 13 weeks, with 6 weeks being the most frequent training duration. Three weekly sessions were conducted in five of the studies. The study protocols ranged from 2 to 9 sets, while 3 sets was the most widely used pattern (Behm, 1991; Rhyu, Kim \& Park, 2015; Thorborg et al., 2016). Most trials used 10 repetitions, while one study performed repetitions to maximal muscle fatigue (Hostler et al., 2001). Regarding the intensity, all studies utilized percentage of the 1RM, with $70-75 \%$ of $1 \mathrm{RM}$ the most representative value; however, Bellar et al. (2011) and Hostler et al. (2001) used between $85 \%$ and $100 \%$ of 1 RM. The rest between sets ranged from 60 to 120 seconds. Unfortunately, no study provided the duration of each session with the exception of Thorborg et al. (2016). The majority of studies compared exercises with elastic resistance and other devices; the most used exercises were the bench press, squat and shoulder press.

\section{Characteristics of the studies in middle-aged adults}

Six studies were included with adults 45 to 64 years old. All studies used healthy pre- and postmenopausal women. 244 women were evaluated: 130 used with elastic bands, 28 used weight machines and 32 exercised in the aquatic environment. The programs lasted between 8 and 24 weeks, with the most common durations being 8 (Heislein, Harris \& Jette, 1994; Thiebaud et al., 2013) and 10 (Colado et al., 2012a; Colado \& Triplett, 2008) weeks. Common parameters included 2-3 sessions per week, 3 sets of 20 repetitions, and rest between sets was 30 seconds. The intensity was generally based on a score of 5 to 7 using the OMNI-RES scale for elastic resistance training (Colado, Triplett, Tella, Saucedo \& Abellán, 2009; Colado et al., 2012a; Colado et al., 2012b), while Winters-Stone \& Snow (2006) used a $60-80 \%$ of the 1RM. Only Colado, Triplett, Tella, Saucedo \& Abellán (2009) and Heislein, Harris \& Jette (1994) provided the total duration of each session, which was 30 to 60 minutes. Full body exercises were used in nearly all the studies, althougth Thiebaud et al. (2013) exclusively used upper limbs exercises.

\section{Characteristics of the studies in older adults}

For older adults (over 64 years old), 32 studies with a total of 1746 subjects were evaluated: 1045 trained with elastic bands and 701 served as a control group. The duration of the training program ranged between 3 and 52 weeks. The most frequently used durations were 8 and 24 weeks; however, 12 weeks were used in six studies. Weekly training frequency ranged from 2 to 4 days. The number of sets ranged between 1 and 4 , and repetitions ranged from 8 to "as many as possible" (Damush \& Damush, 1999). Nine studies utilized 10 repetitions per set; unfortunately, 6 trials did not provide repetition information. The least reported parameter in this age group was intensity, with 19 studies (59\%) not providing this information. Seven studies used scales of perceived effort to prescribe intensity (five used the Borg's scale and 2 used the OMNI-RES for elastic bands). Four studies used the $\%$ of the 1RM, and Hofmann et al. (2016) prescribed intensity based on the the progressive increase in resistance based on the different colors of the elastic bands. The rest time between exercises was only provided in five studies, ranging between 30 and 120 seconds. Most studies used a 60 minute session duration, although 7 trials did not report this information. 13 studies used multi-joint exercises such as squats, lunges, lat pull down or horizontal rows, among others. 16 trials did not mention the specific exercises that were used, providing only the number of exercises performed in the session. Two articles were exclusively focused on the lower limbs (Kwak, Kim \& Lee, 2016; Yasuda et al., 2016), while one study 
only used in upper limbs (Yasuda et al., 2014). Both studies of Yasuda et al. (2014, 2016) used blood flow restriction using elastic cuffs.

\section{Methodological quality of included trials}

After grading the individual study quality using the PEDro scale, the average data obtained in studies among each age group was: (a) Youths: 5 points on average (High quality: 1 Study; Low Quality: 2 studies). (b) Young Adults: 4.66 points on avesrage (High quality: 2 studies; Low Quality: 4 studies). (c) Middleaged adults: 4.83 points on average (High quality: 2 studies; Low Quality: 4 studies). (d) Older Adults: 4.68 points on average (High quality: 10 studies; Low Quality: 22 studies).

A majority of studies in each group scored below 6 on the PEDro scale, indicating low methodological quality. Table 1 shows the specific PEDro scores of each article.

\section{Discussion}

This systematic review suggests that elastic resistance training programs are effective to improve muscle strength, physical function and other healthrelated variables across the lifespan. These data expand the current evidence on the effectiveness of these exercise programs among adults (de Oliveira et al., 2016) and elderly participantants (Martins et al., 2013). Elastic resistance programs improved the functional capacity of young, middle, and older adults (Colado, Triplett, Tella, Saucedo \& Abellán, 2009; Cyarto, Brown, Marshall \& Trost, 2008; Franzke et al., 2015) as well as body composition (Colado, Triplett, Tella, Saucedo \& Abellán, 2009; Lubans, Aguiar \& Callister, 2010) in all ages, except in the oldest adults, where the results were mixed (Skelton, Young, Greig \& Malbut ,1995; So et al., 2013). Interestingly, some recent studies have demonstrated positive health effects in 80 year olds (Chupel et al., 2017; Furtado et al., 2019; Rieping et al., 2019). Finally, there were few or irrelevant results on health biomarkers after elastic resistance training (Hostler et al., 2001; Lubans, Mundey, Lubans \& Lonsdale, 2013).

\section{Effects of training with elastic bands in youths}

An important finding among elastic resistance training studies in youths was improvement in the motivation and participation of the child (Barkley, Ryan, Bellar, Bliss \& Roemmich, 2011), possiblybecause it seems more attractive and provides more security than heavy weigths (Annesi, Westcott, Faigenbaum \& Unruh, 2005). In addition, elastic resistance training has shown greater physicalperformanceimprovements than bodyweight exercises (Coskun \& Sahin, 2014). Most likely, the stimulus provided by the bodyweight exercises was not enough or the exercise was too difficult to adapt to each subject (Faingenbaum, 2000), while the elastic band resistance could be easily adapated, thus providing a proper stimulus for the neuromuscular improvements (Ignjatović, Stanković, Radovanović, Marković \& Cvećka, 2009). Studies of Lubans, Aguiar \& Callister (2010) and Sahin, Aslan \& Demir (2016) found improvements in strength similar or higher to free weights training. For body composition, Lubans, Aguiar \& Callister (2010) found changes in the percentage of fat-free and fat mass in both sexes, although the bodyweight training group achieved greater results than the elastic resistance group. It is possible that suboptimal dosing of intensity, duration, and frequency of elastic resistance training may provide a low stimulus for improving body composition due to the suboptimal intensity, short duration and frequency of the protocols used. It is also interesting that some investigations were focused on the effects of elastic resistance training in children with special needssuch as cerebral palsy (Shin \& Kim, 2016). Finally, it's important to note that no study provided data on functional capacity or health biomarkers, thus leaving a need for further investigation.

Effects of training with elastic bands in young adults

The majority of the studies including young adults were focused on evaluating the effects of elastic resistance training on muscle strength. Bellar et al. (2011) obtained better results by adding elastic bands to a bench press compared to free weights alone; this combination was more effective at improving muscle strength than the isolated use of free weights, even in inexperienced subjects. These results may be due to a mechanical change created by using elastic resistance that helps to overcome the "sticking point" during the concentric phase of the movement, possibly facilitating neural adaptations (Kompf \& Arandjelovic, 2016). Hostler et al. (2001) reported an $\mathrm{RM}$ increase in the squat with no change in the number of repetitions during knee extension. Behm (1991) analyzed the effect of different devices (i. e. free weights, aquatic resistance and elastic resistance) finding a similar response in muscle strength between them. Sugimoto \& Blanpied (2006) analyzed the 
effect of elastic band exercise of the shoulder rotator cuff; they found that the elastic resistance provided greater internal and external rotator strength than a flexible foil (Bodyblade). Thorborg et al. (2016) observed an improvement in isometric hip flexion strength after 6 weeks of elastic resistance training. Some researchers have combined elastic resistance with proprioceptive neuromuscular facilitation (PNF) techniques, reporting improvements in joint mobility, reporting benefits in the development of the strength, and the balance through of improving the responsiveness of the skeletal-muscle system (Jonghwan, Chulhyun, Kyumoon, Hyeonju \& Byeungok, 2003; Rhyu, Kim \& Park, 2015). Only Hostler et al. (2001) analyzed the effect of elastic resistance exercise on some physiological biomarkers of health, finding no significant improvements. No research has been published on body composition and functional capacity outcomes in young adults using elastic resistance training.

Effects of training with elastic bands in middle-aged adults

The majority of the studies including middle-aged adults evaluated the effects of elastic resistance training on on functional capacity. Colado \& Triplett (2008), Colado et al. (2009) and Colado et al. (2012b) analyzed functional capacity, reporting significant increases after the intervention. Thiebaud et al. (2013) observed that two training groups (low and mediumhigh intensity) increased their 1RM in upper and lower limb exercises. Heislein et al. (1994) reported 20 and $8.8 \%$ increases in quadriceps and hamstrings strength respectively, using elastic bands together with PNF techniques similar to Rhyu et al. (2016). Five studies reported improvements after elastic resistance training interventions on body composition, measured with bioelectrical impedance analysis (Colado \& Triplett, 2008; Colado, Triplett, Tella, Saucedo \& Abellán, 2009; Colado et al., 2012a; Winters-Stone \& Snow, 2006) or musculoskeletal ultrasound (Thiebaud et al., 2013). Colado \& Triplett (2008), Colado, Triplett, Tella, Saucedo \& Abellán (2009) and Colado et al. (2012a) reported more improvemnets in body composition using elastic bands than compared to other resistance devices. They indicated that a similar routine during a short-term training program is sufficient to reduce the percentage of fat mass, regardless of the type of resistance. Winter-Stone \& Snow (2006) assessed bone mineral density after a lower and/ or upper body elastic resistance program that was complemented with jump exercises, demostrating significant improvement in the greater trochanter, probably because the skeletal system needs high load exercises to maintain or develop their levels of density (Iwamoto, 2013). Only Colado, Triplett, Tella, Saucedo \& Abellán (2009) and Flandez et al. (2017) analyzed health-related biomarkers, showing improvement both metabolic health as well as motor function. Colado, Triplett, Tella, Saucedo \& Abellán (2009) also showed a reduction in diastolic blood pressure with a medium-term elastic resistance training program. Importantly, the majority of these changes exposed were found in only short and medium-term programs; therefore, it seems logical that longer programs would provide greater results (Braith \& Stewart, 2006), although more research is needed.

Effects of training with elastic bands in older adults

In 23 studies of older adults exercising with elastic resistance, functional capacity was the most analyzed variable, reporting improvements of 2025\%. 15 studies reported strength improvements of $15-20 \%$, either in the lower limbs or upper limbs. Eight studies investigated the effect of elastic resistance training on body composition; only 3 found significant improvements. 12 trials carried out measurements on health-related biomarkers using blood tests, hormonal levels, enzyme activity or oxidative stress. Interestingly, chromosomal damage tended to decrease after elastic resisatnce training (Franzke et al., 2015a and b). Hofmann et al. (2016) showed a decrease in the percentage of myostatin and increases in follistatin, but no change in the hormone IGF-1. Park et al. (2016) found a 14\% improvement in systolic blood pressure, while Lubans, Aguiar \& Callister (2010) reported a tendency to improve. Aniansson, Ljungberg, Rundgren \& Wetterqvist (1984) revealed improvements of up to $39 \%$ in the myokinases and $44 \%$ in the citrate synthase after 40 weeks of exercising with elastic bands and with the own body weight. Lastly, inconsistent data was observed for hemodynamic parameters, such as blood pressure, cardiac output, ejection volume, or heart rate, likely due to a lack of appropriate training stimulus (Vincent, Vincent, Braith, Bhatnagar \& Lowenthal, 2003) as was the case in the majority of studies included in this age group.

It appears that elastic resistance training in older adults is beneficial either in healthy subjects or those with pathology. Older adults consistently experience strength gains with elastic resistance training programs (Capodaglio et al., 2002; Martins et al., 2013; Martins et al., 2015; Oh et al., 2016). These 
findings support the benefits of strength training with elastic resistance to prevent and treat dynapenic and/or sarcopenic processes associated with age (Clark \& Manini, 2008; Rosenberg, 1997).

\section{Limitations of the study}

Based on data from the included studies, the present systematic review provides novel data about evidence on the effectiveness of elastic resistance training programs across the lifespan. However, the small number of articles using elastic resistance training (especially at the youngest ages) and the general poor quality of the studies are the main limitations of this study. Because of these limitations, and due to the heterogeneity of training parameters between studies, a dose-response relationship can not be provided from these data. In addition, the lack of information about the dose or exercises used in some studies, especially among elderly subjects, limits the ability to provide reccommendations.

High-quality dose-response clinical trials are needed to better understand the optimal dose and the effectiveness of these programs at different ages, especially in children. More research is needed on the effects of training with elastic resistance on healthrelated biomarkers and body composition variables. Future studies should clearly describe the training volume, intensity, frequency, duration and rest intervals used in the exercise programs. Until then, practitioners should follow standard strength training parameters in elastic resistance programs.

Finally, this review included only articles published before March 2017 due to the lengthy time that it was needed for editing, and also due to the usual time needed for beeing reviewed and accepted to definitive publication in a high quality scientific journal.

\section{Conclusions}

Based on this systematic review, training with elastic resistance improves muscle strength and functional capacity across the lifespan. These results suggest that elastic resistance benefits are similar to those seen with traditional isotonic machines and free weights. In addition, elastic training seems to be effective in improving body composition and may have positive results in some health biomarkers, although further research is needed.

\section{Funding}

This work was supported by the grant Ignacio Larramendi 2014 from the Mapfre Foundation (Code: OTR2015-140931NVES). Pedro Gargallo was supported by a predoctoral scholarship (FPU15/ 05634) awarded by the Spanish Ministry of Education, Culture, and Sport. 


\begin{tabular}{|c|c|c|c|c|c|c|c|}
\hline & 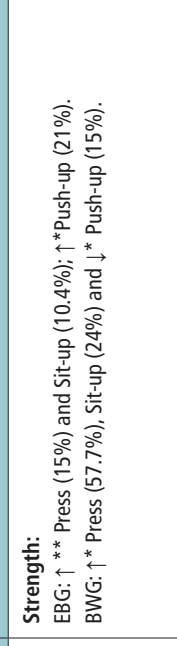 & 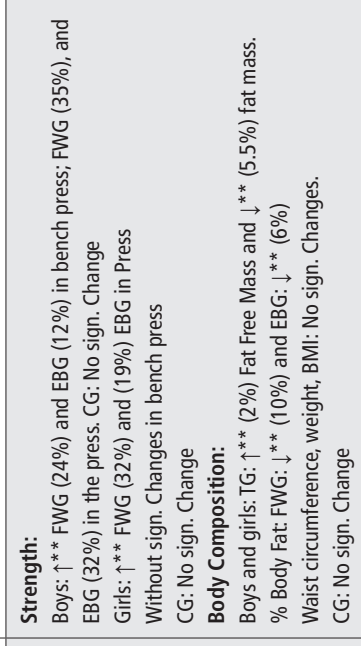 & 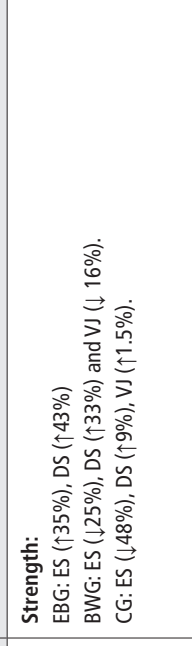 & & 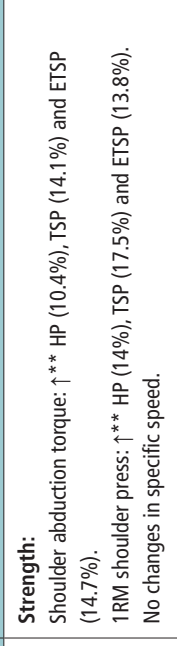 & 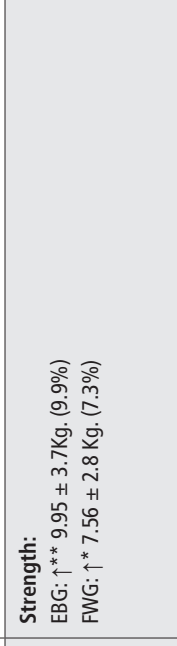 & 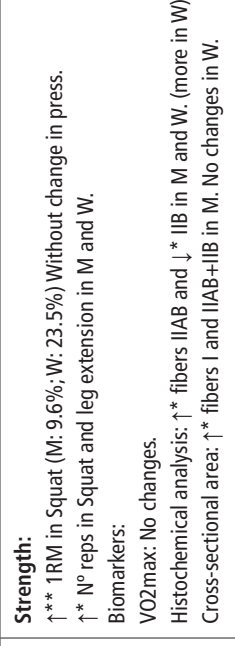 \\
\hline 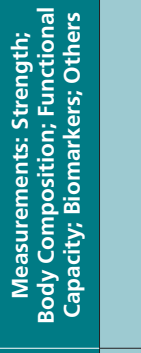 & 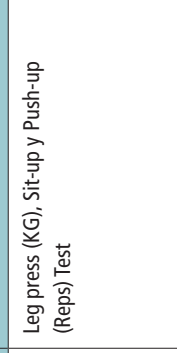 & 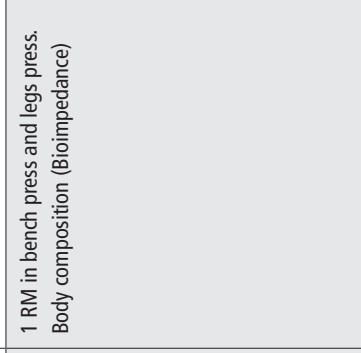 & 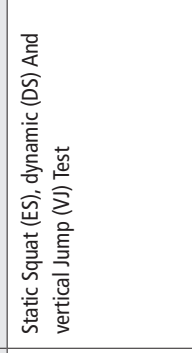 & & 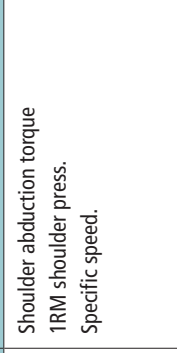 & 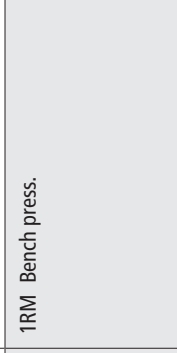 & 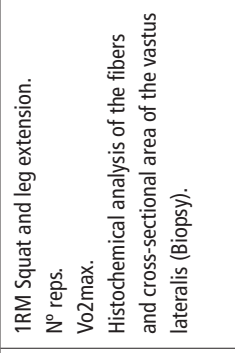 \\
\hline 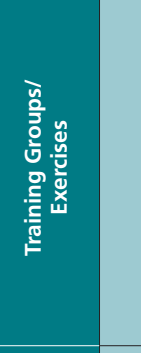 & 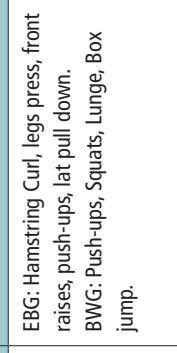 & 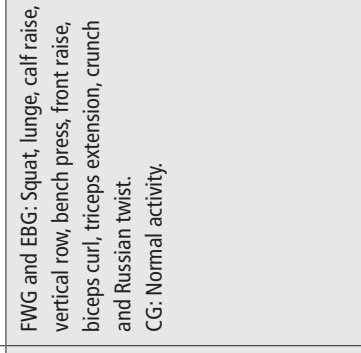 & 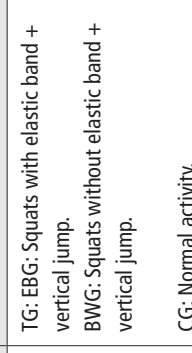 & & 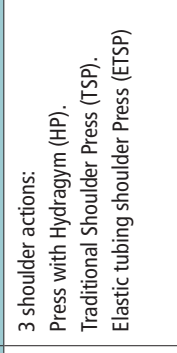 & 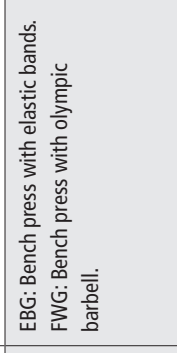 & 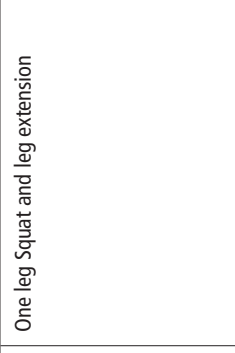 \\
\hline 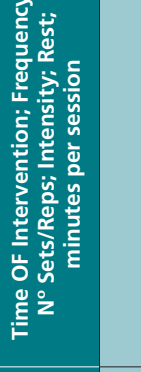 & 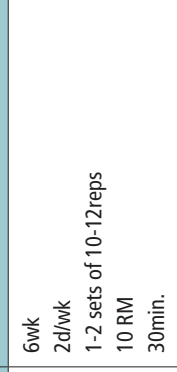 & 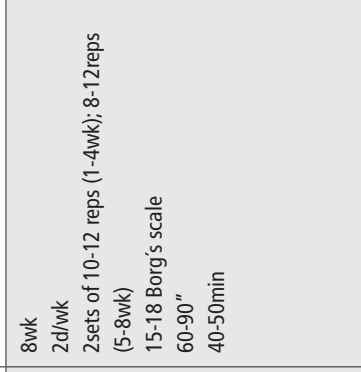 & 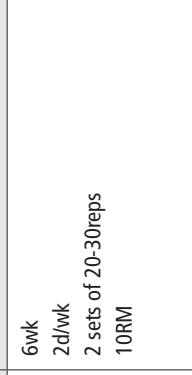 & & 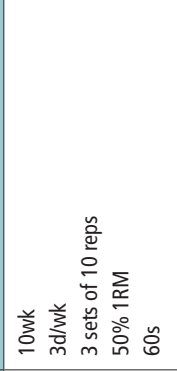 & 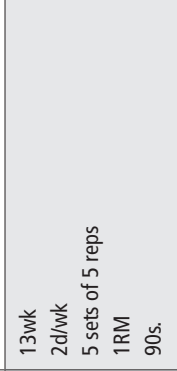 & 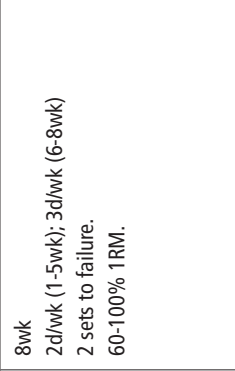 \\
\hline 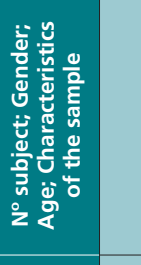 & 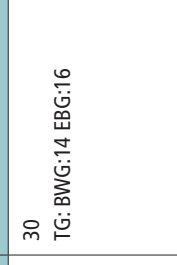 & 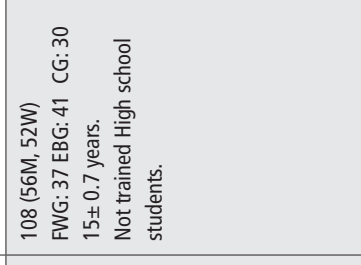 & 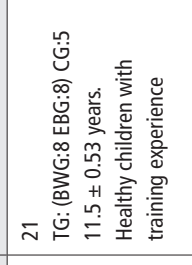 & & 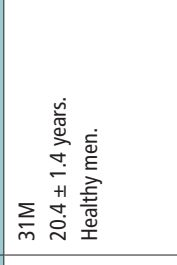 & 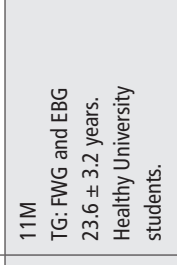 & 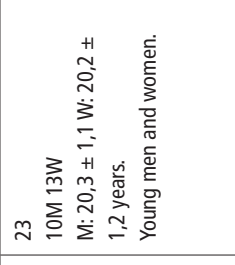 \\
\hline 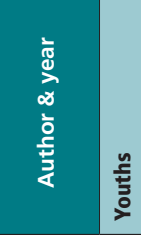 & 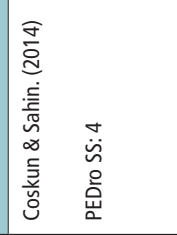 & 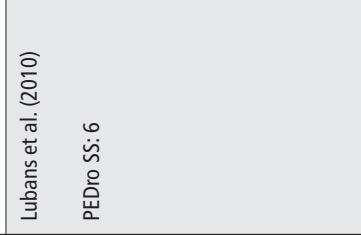 & 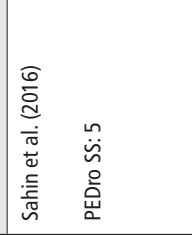 & 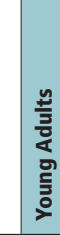 & 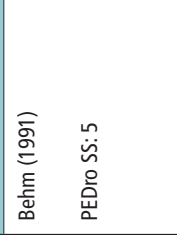 & 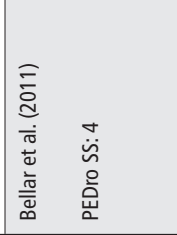 & 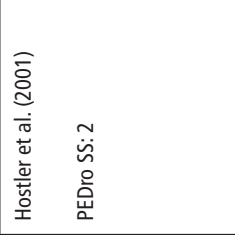 \\
\hline
\end{tabular}




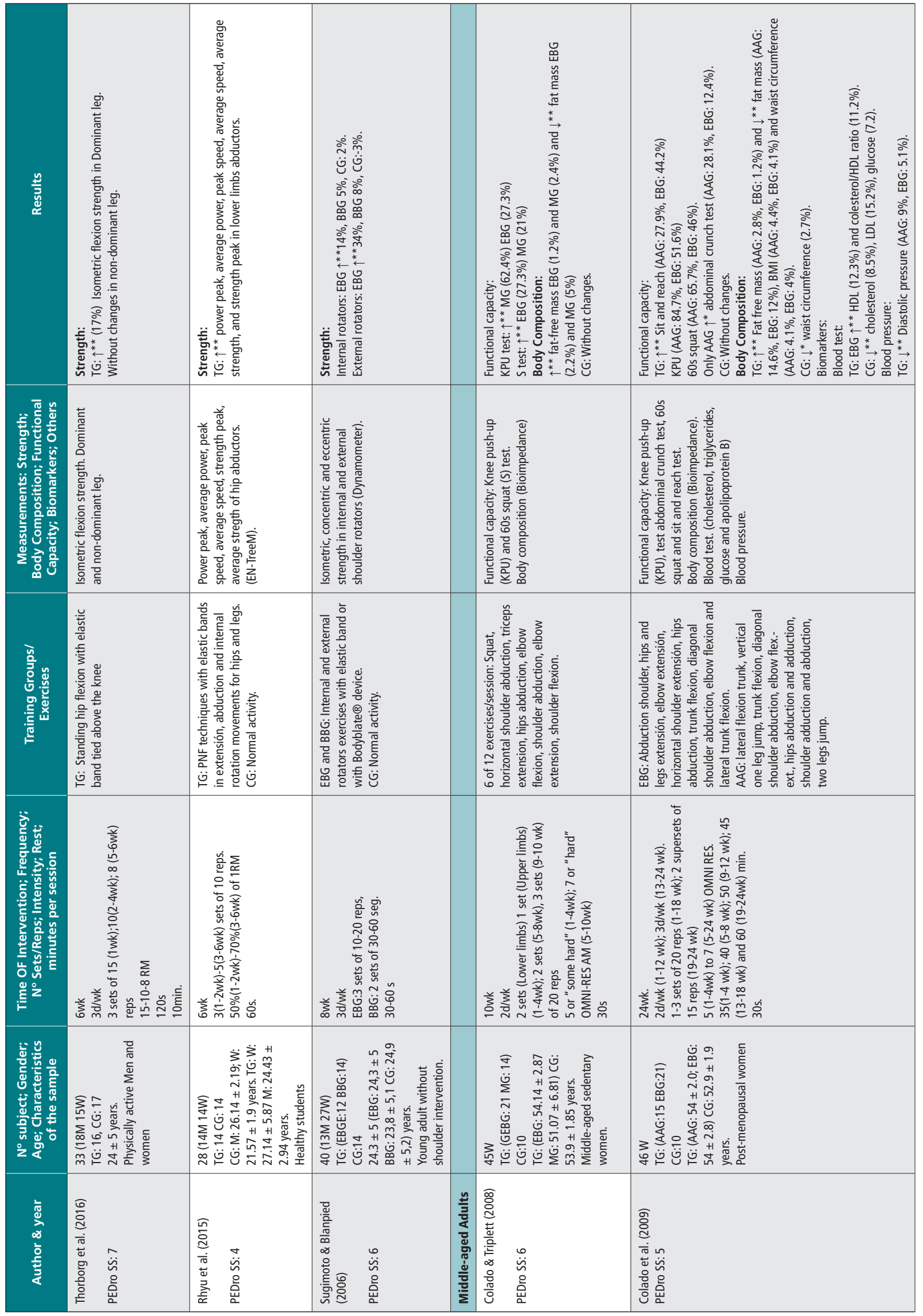




\begin{tabular}{|c|c|c|c|c|c|c|}
\hline & 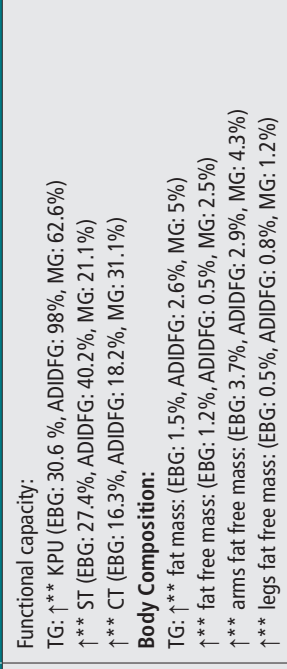 & 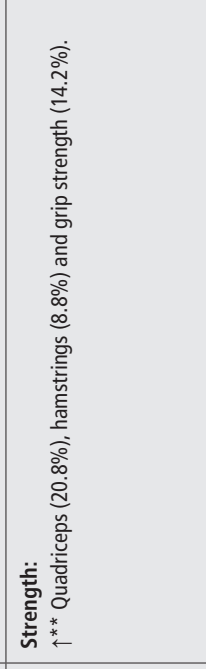 & 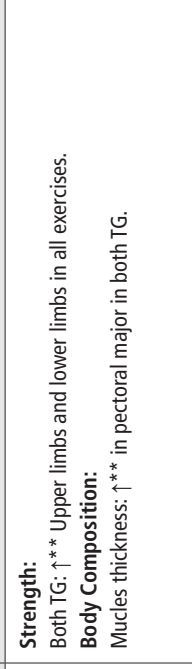 & 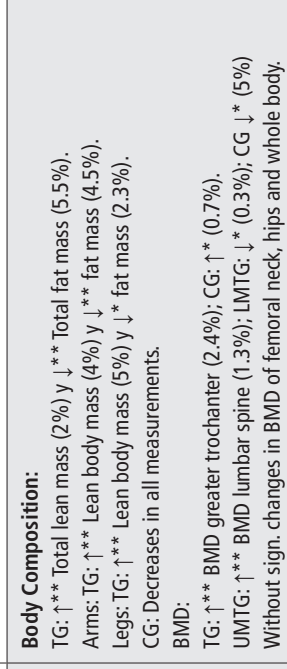 & 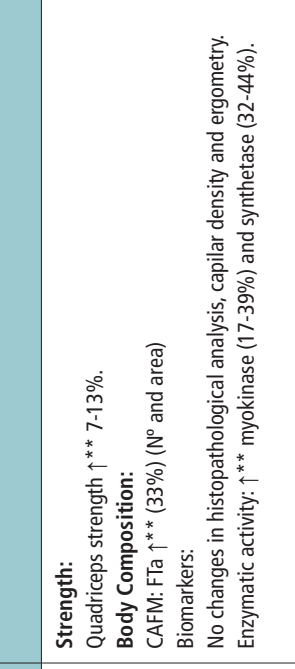 & 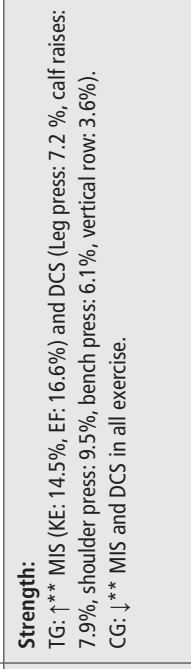 \\
\hline 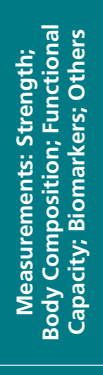 & 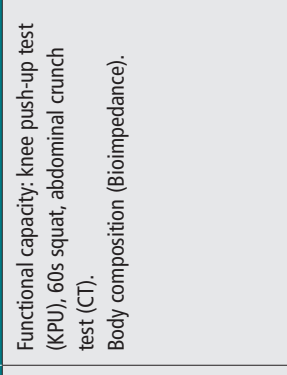 & 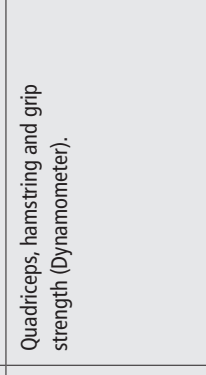 & 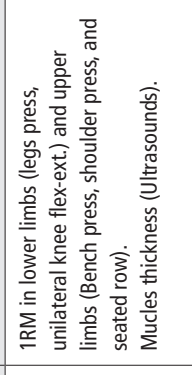 & 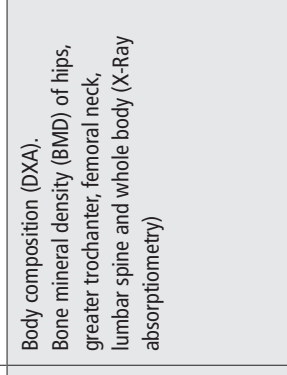 & 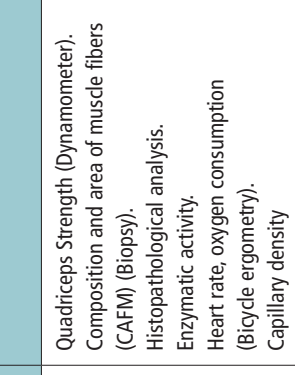 & 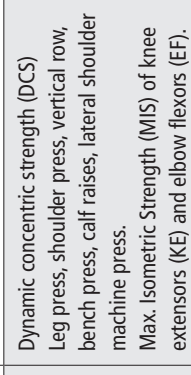 \\
\hline 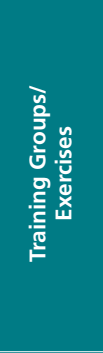 & 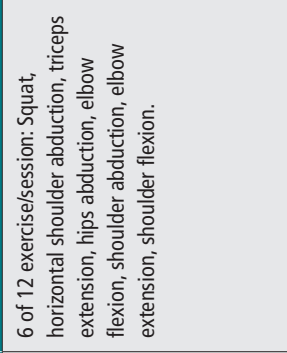 & 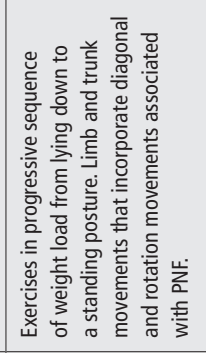 & 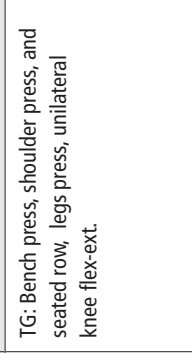 & 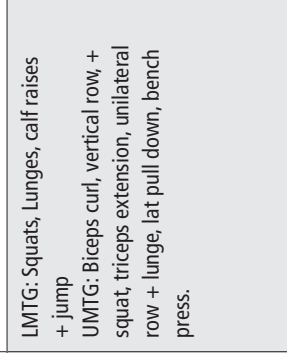 & 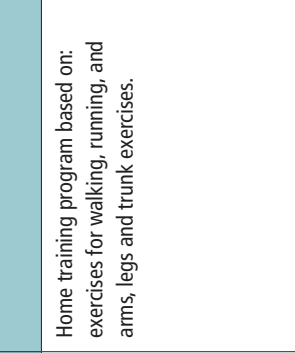 & 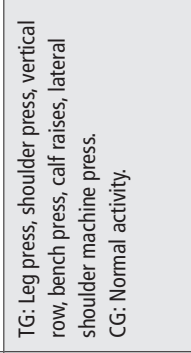 \\
\hline 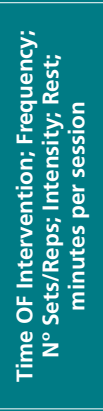 & 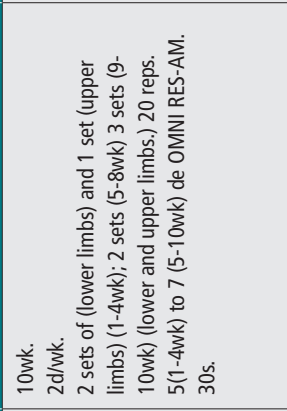 & 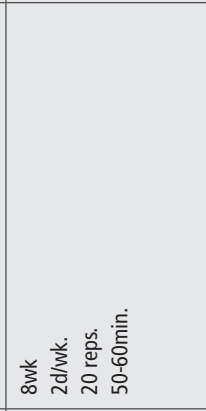 & 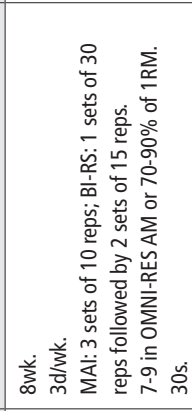 & 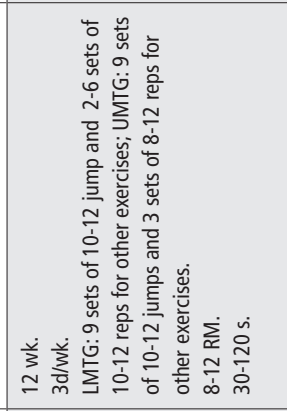 & 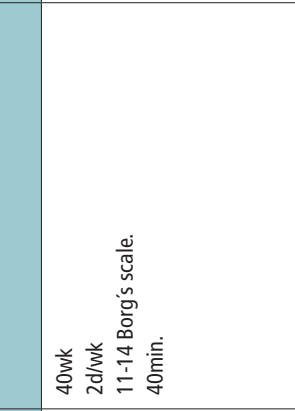 & 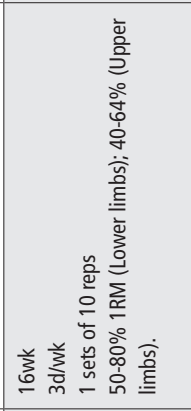 \\
\hline 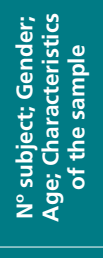 & 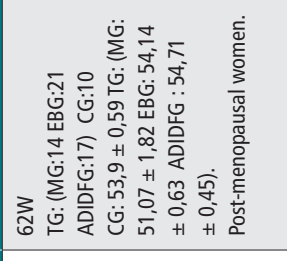 & 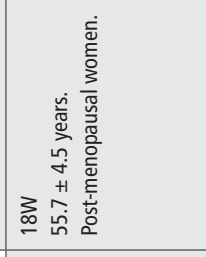 & 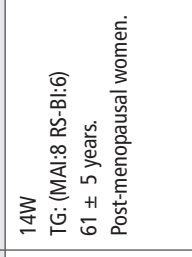 & 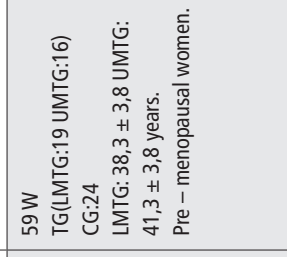 & 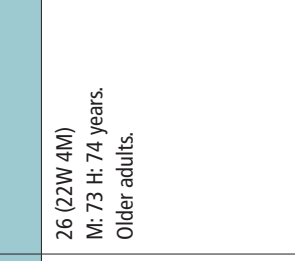 & 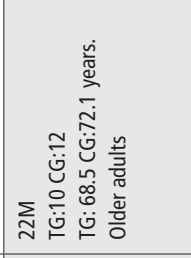 \\
\hline 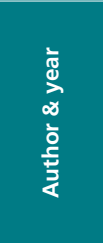 & 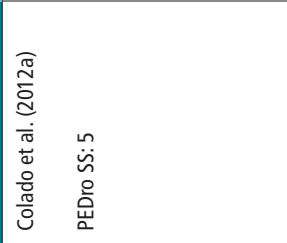 & 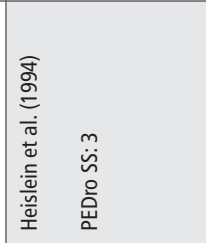 & 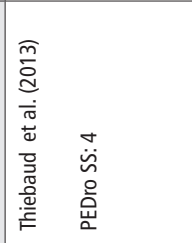 & 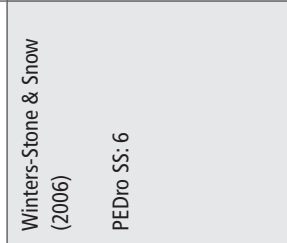 & 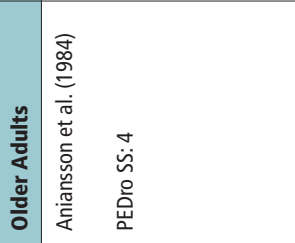 & 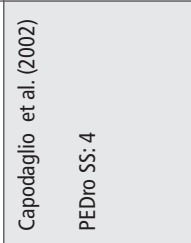 \\
\hline
\end{tabular}




\begin{tabular}{|c|c|c|c|c|c|c|}
\hline & 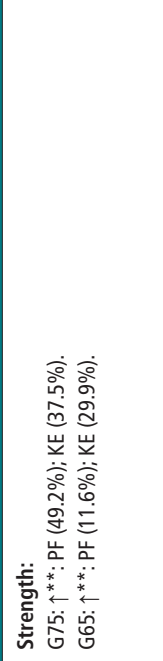 & 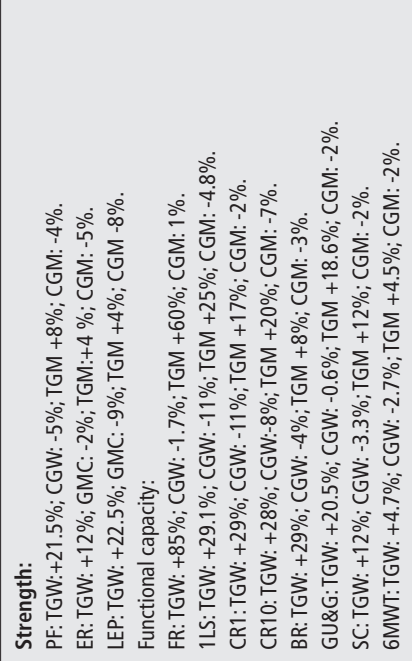 & 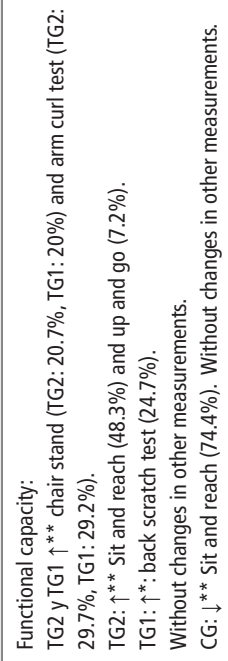 & 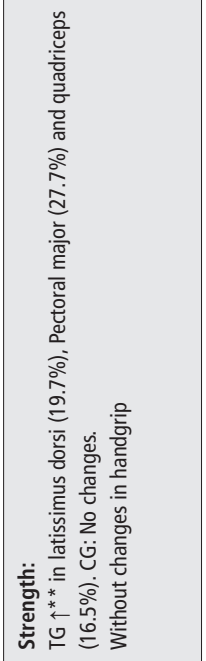 & 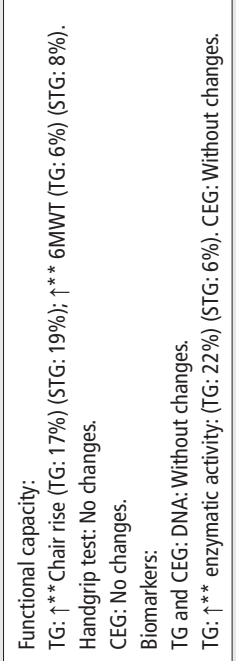 & 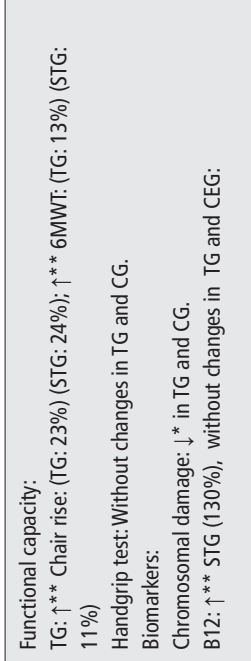 \\
\hline 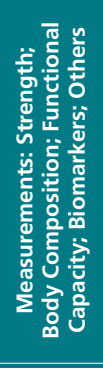 & 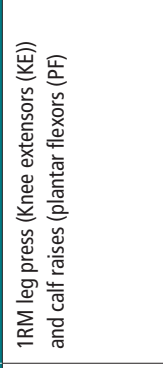 & 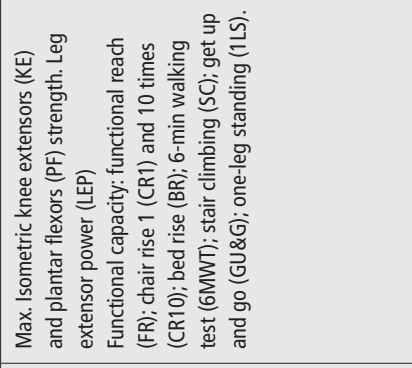 & 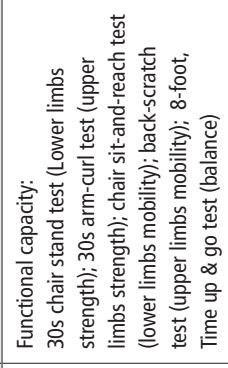 & 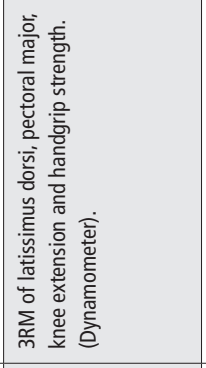 & 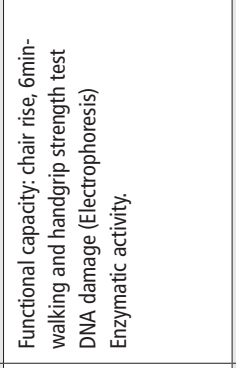 & 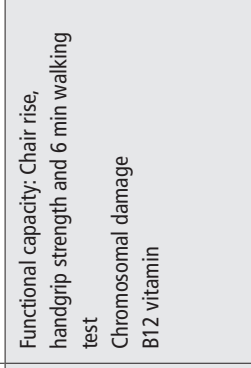 \\
\hline 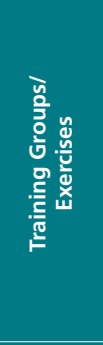 & 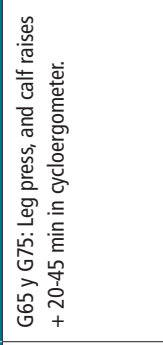 & 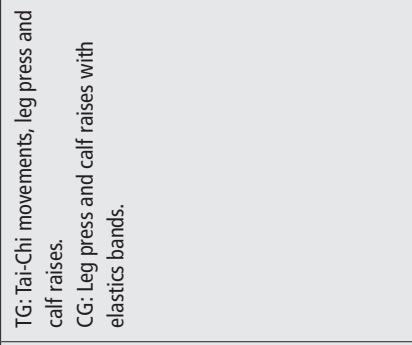 & 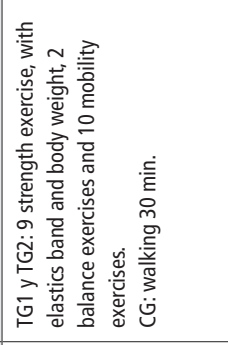 & 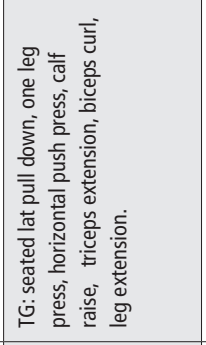 & 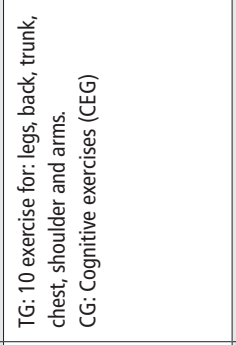 & 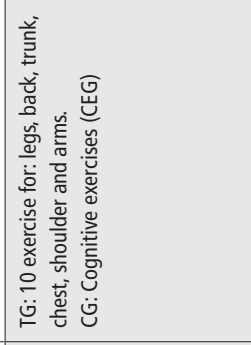 \\
\hline 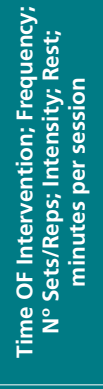 & 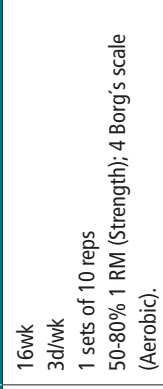 & 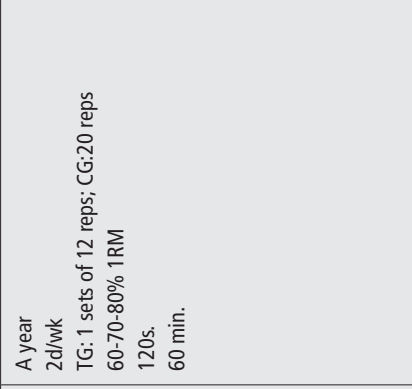 & 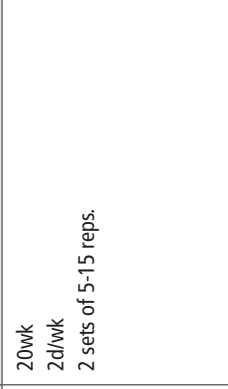 & 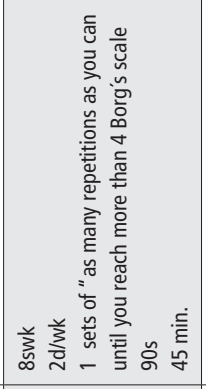 & 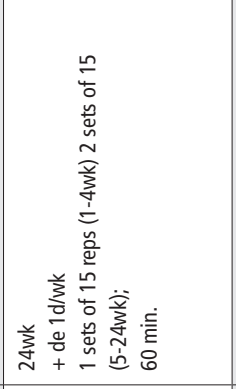 & 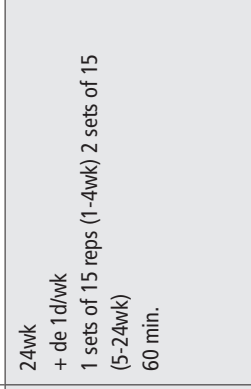 \\
\hline 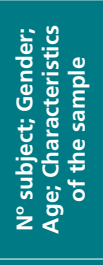 & 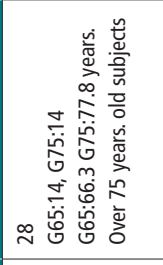 & 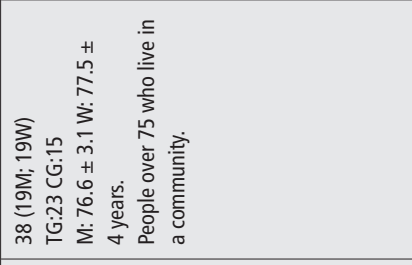 & 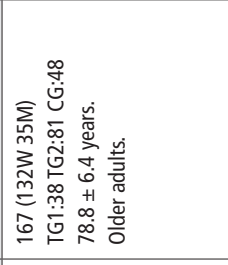 & 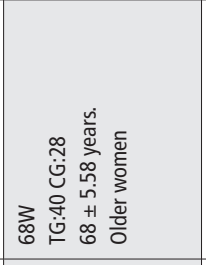 & 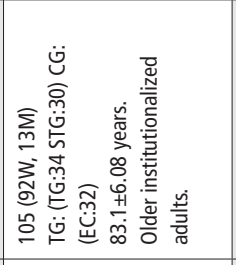 & 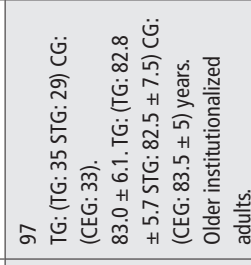 \\
\hline 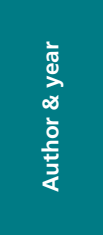 & 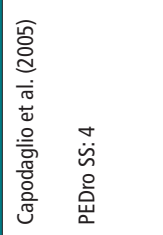 & 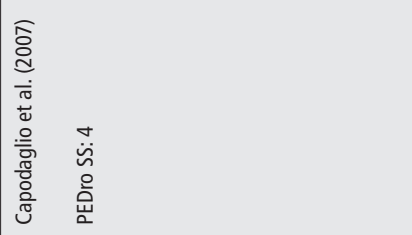 & 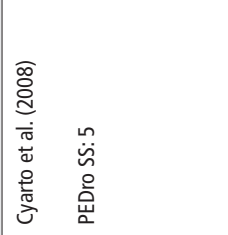 & 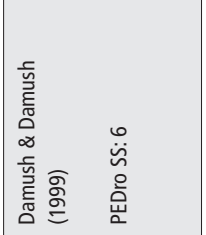 & 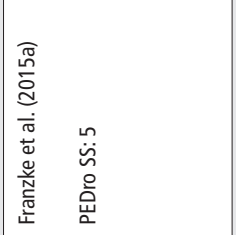 & 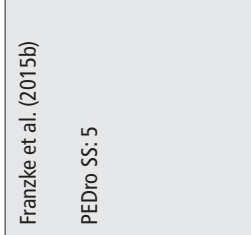 \\
\hline
\end{tabular}




\begin{tabular}{|c|c|c|c|c|c|c|c|c|}
\hline & 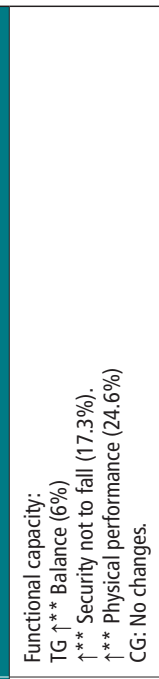 & 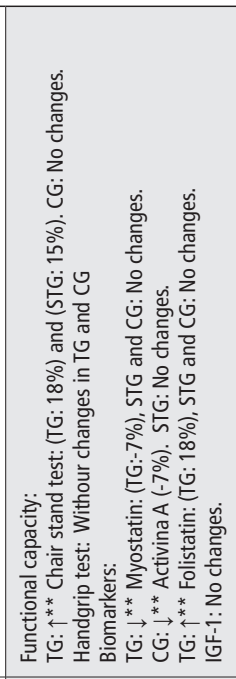 & 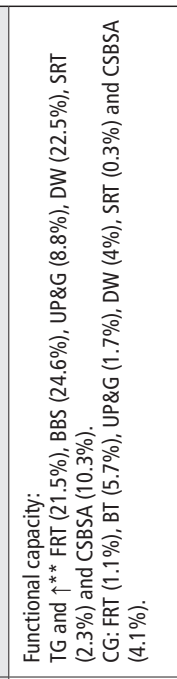 & 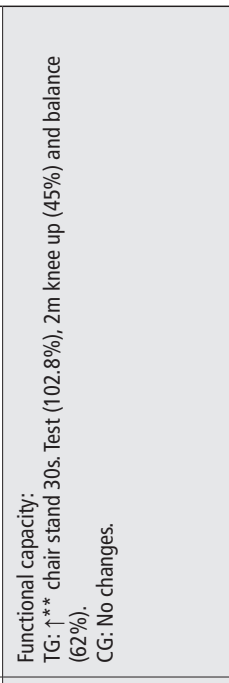 & 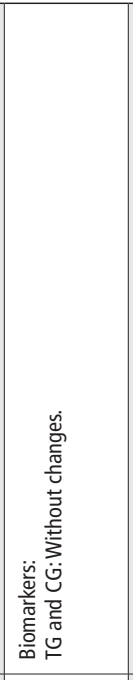 & 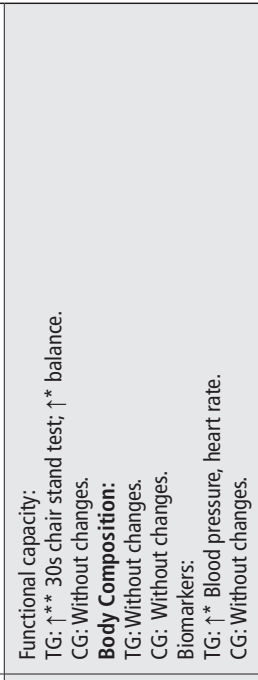 & 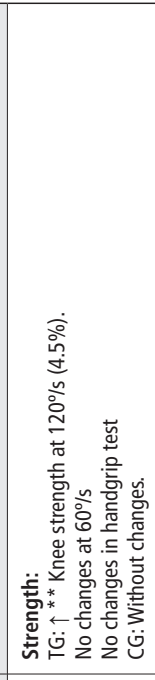 & 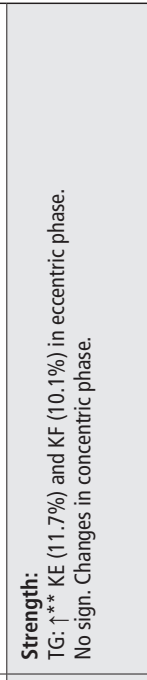 \\
\hline 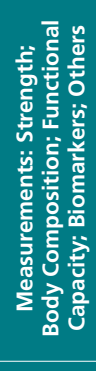 & 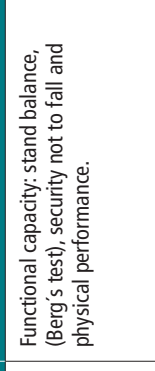 & 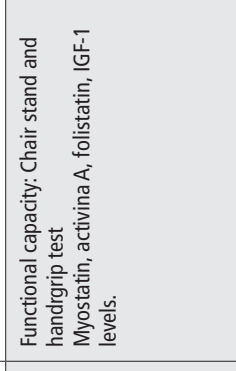 & 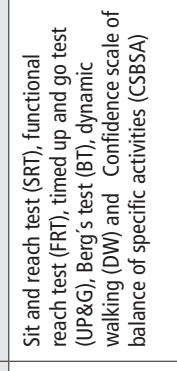 & 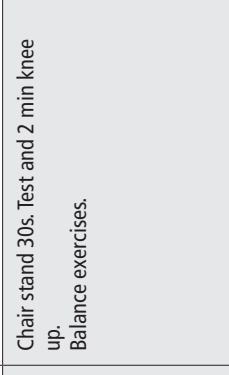 & 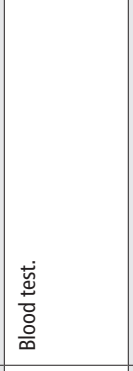 & 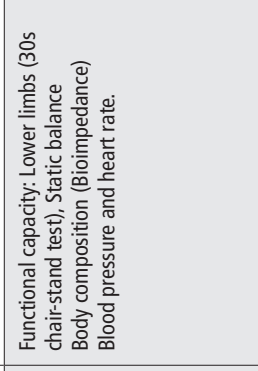 & 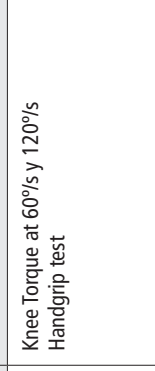 & 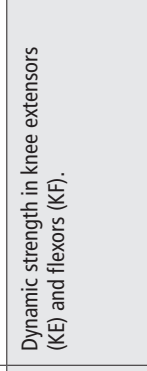 \\
\hline 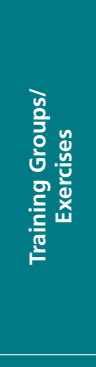 & 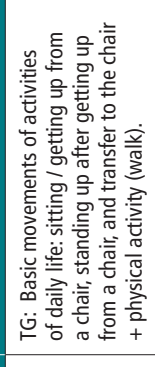 & 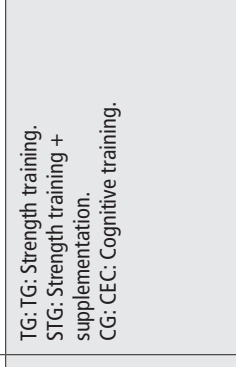 & 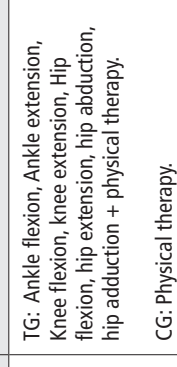 & 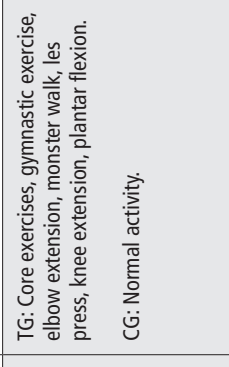 & 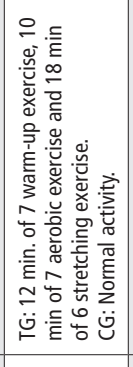 & 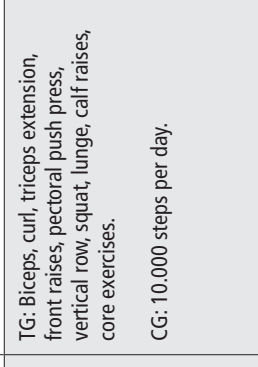 & 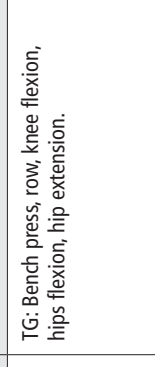 & 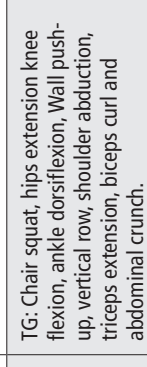 \\
\hline 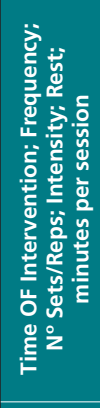 & 总圽言 & 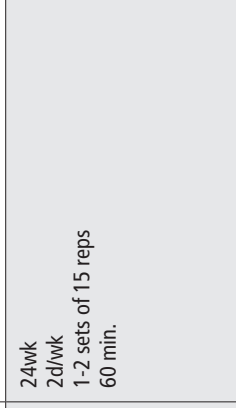 & 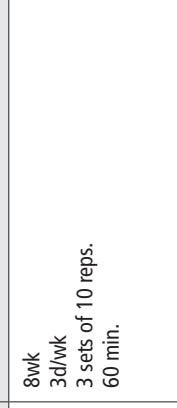 & 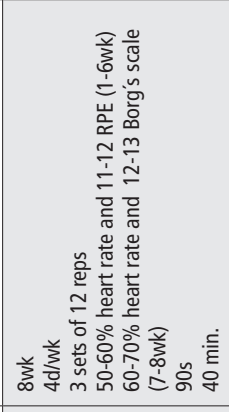 & 美荡言 & 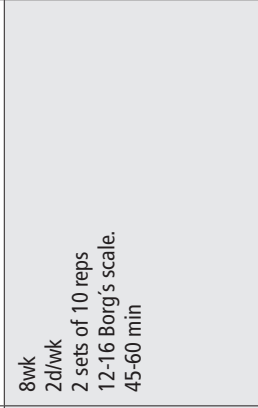 & 恙 & 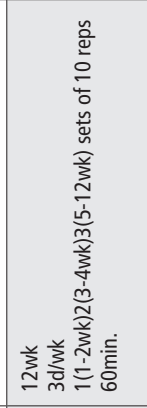 \\
\hline 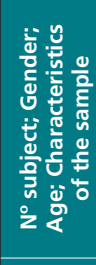 & 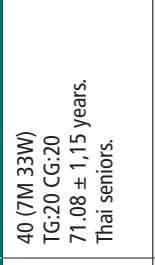 & 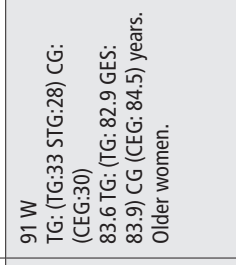 & 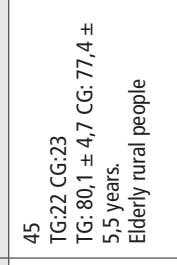 & 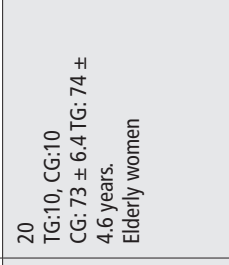 & 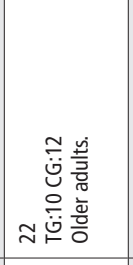 & 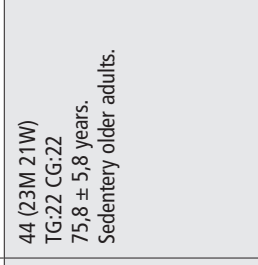 & 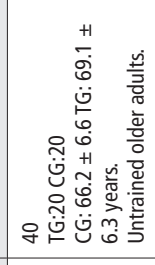 & 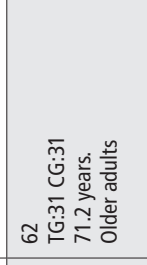 \\
\hline 高 & 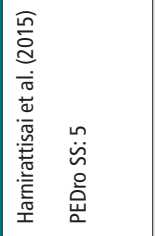 & 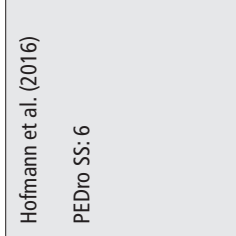 & 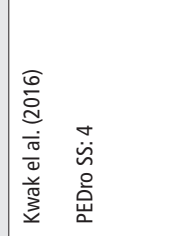 & 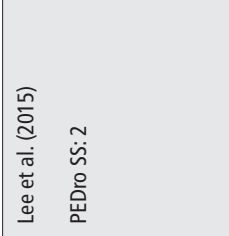 & 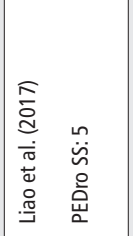 & 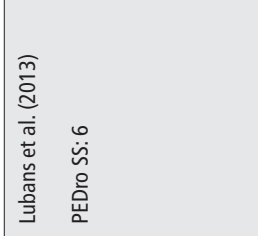 & 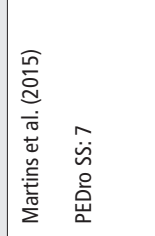 & 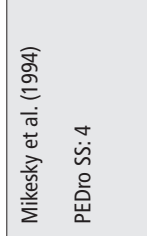 \\
\hline
\end{tabular}




\begin{tabular}{|c|c|c|c|c|c|}
\hline$\frac{\underline{n}}{\bar{z}}$ & 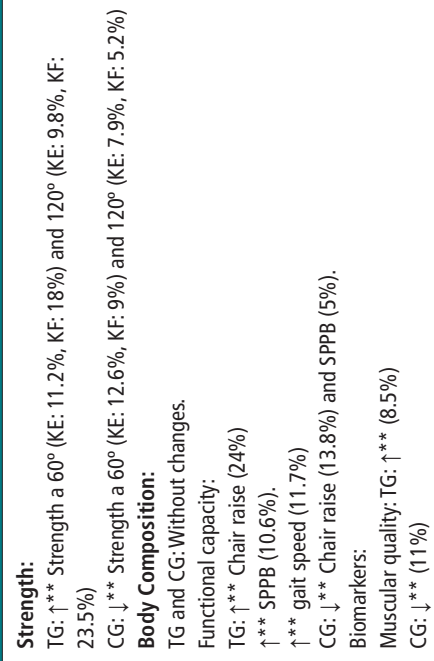 & 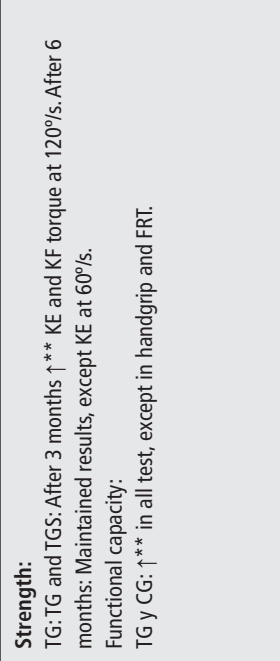 & 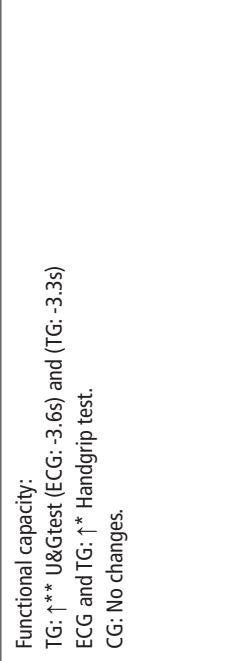 & 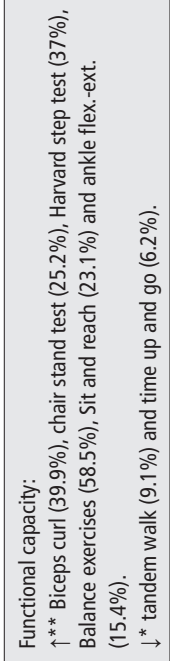 & 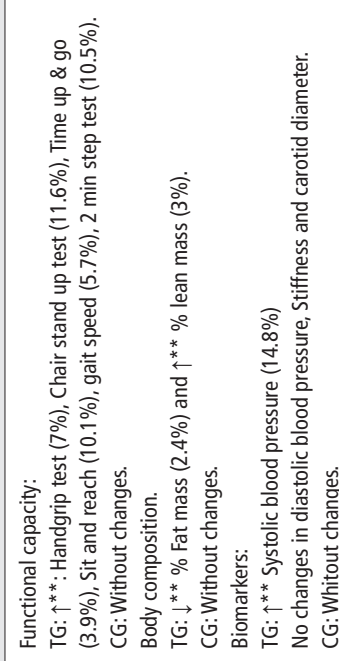 \\
\hline 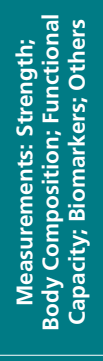 & 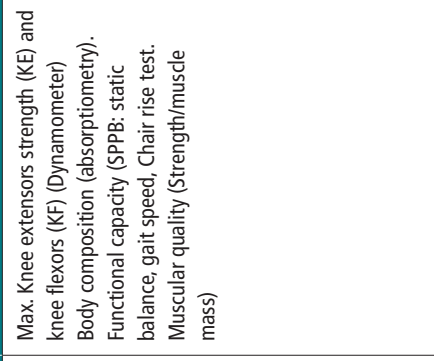 & 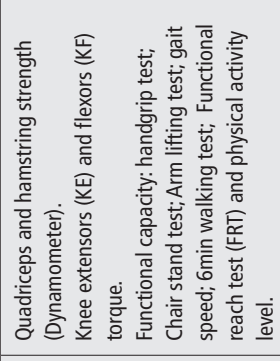 & 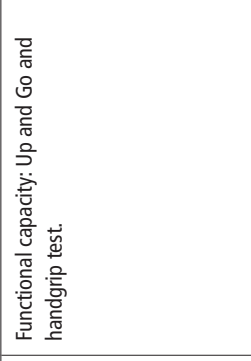 & 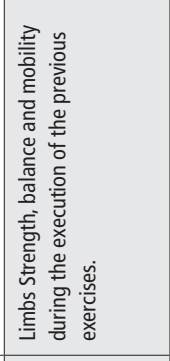 & 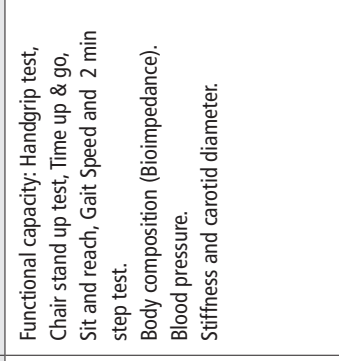 \\
\hline 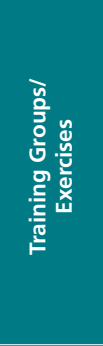 & 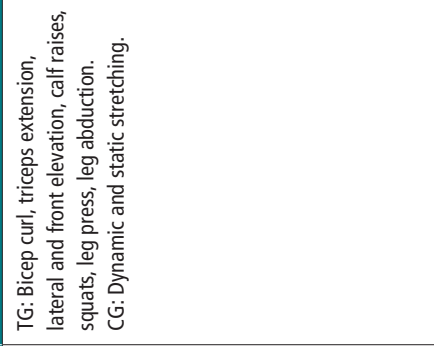 & 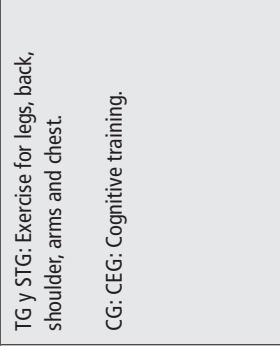 & 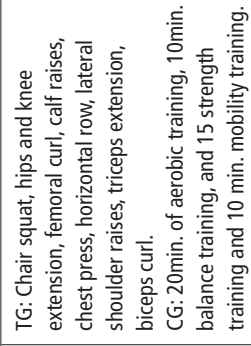 & 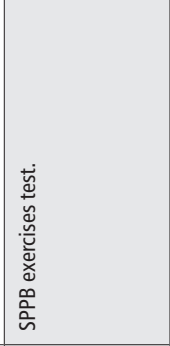 & 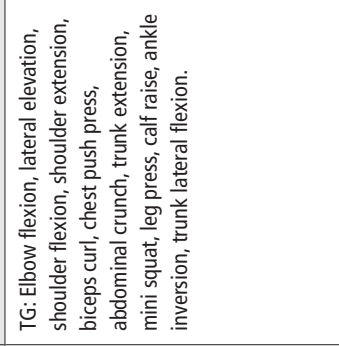 \\
\hline 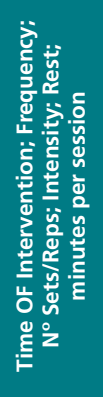 & 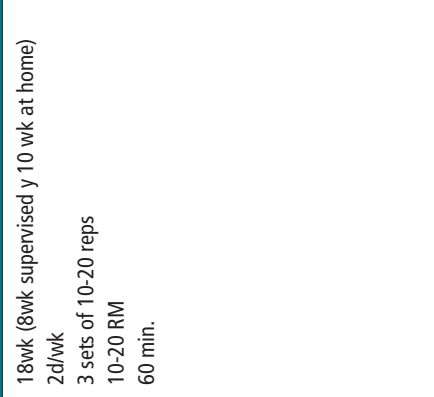 & 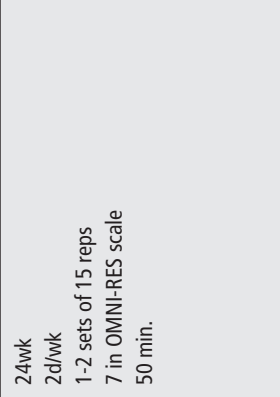 & 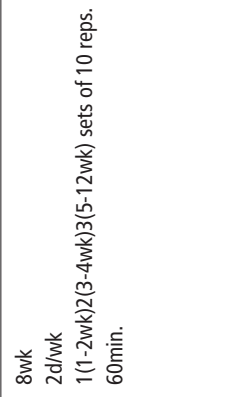 & 恙䇏 & 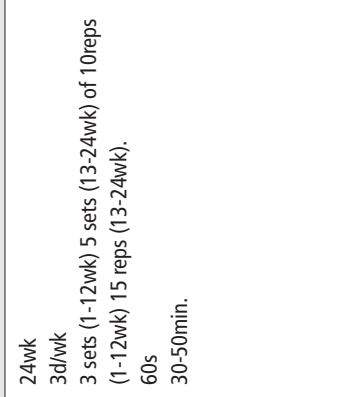 \\
\hline 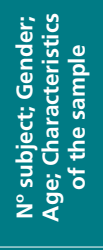 & 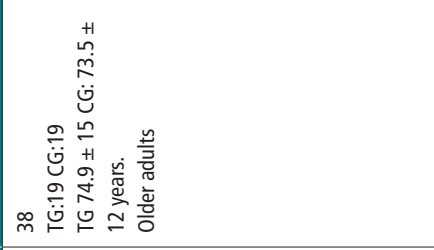 & 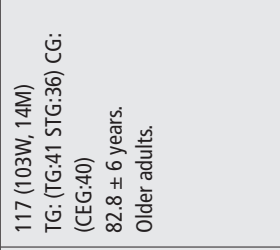 & 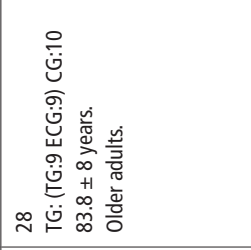 & 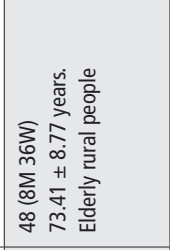 & 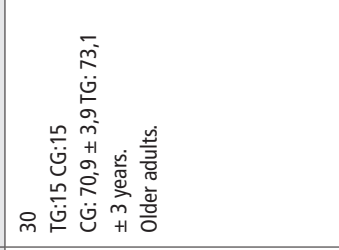 \\
\hline 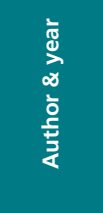 & 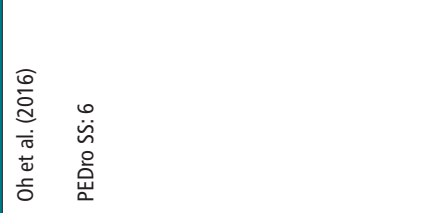 & 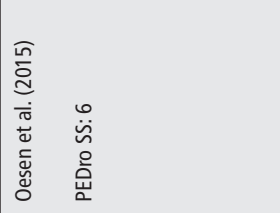 & 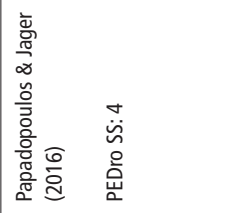 & 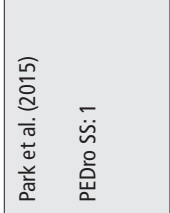 & 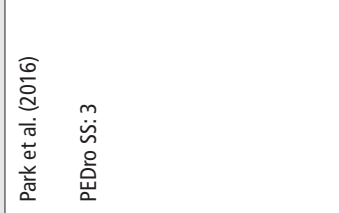 \\
\hline
\end{tabular}




\begin{tabular}{|c|c|c|c|c|c|c|}
\hline 鸹 & 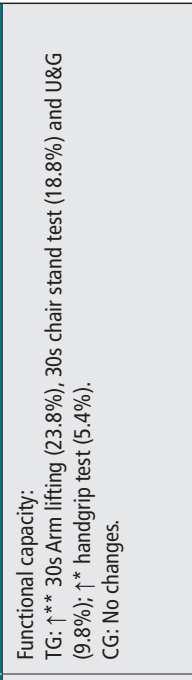 & 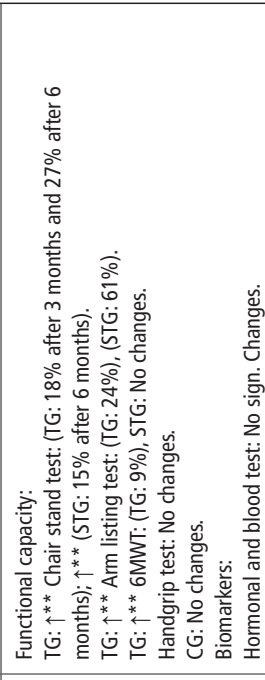 & 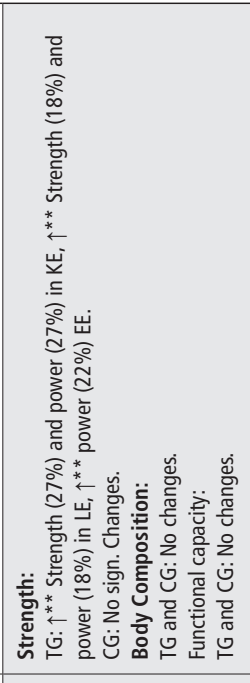 & 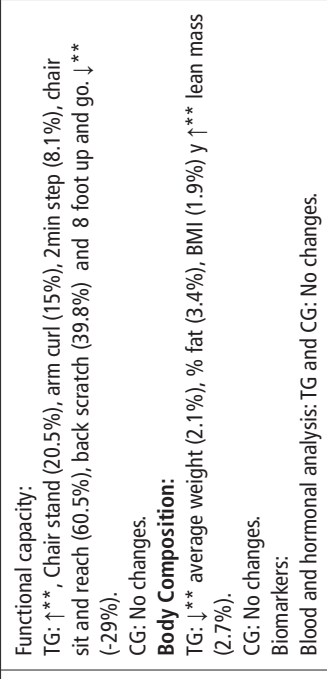 & 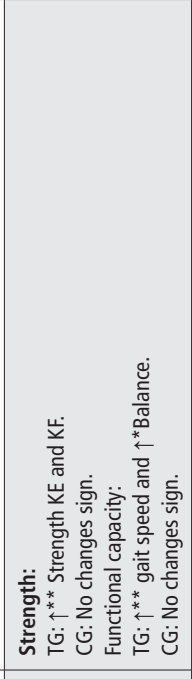 & 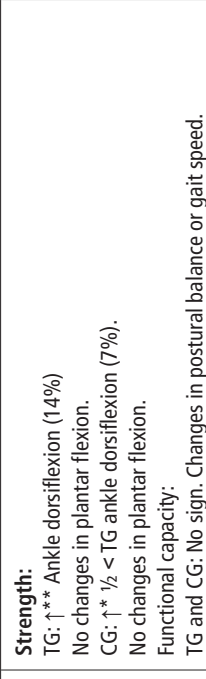 \\
\hline 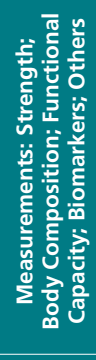 & 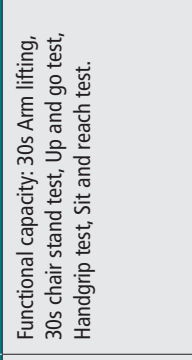 & 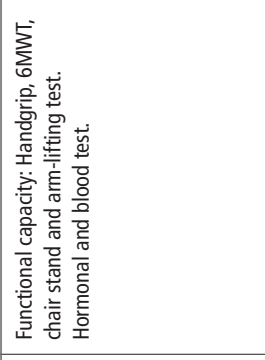 & 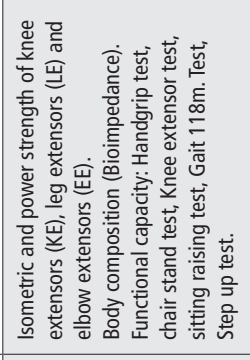 & 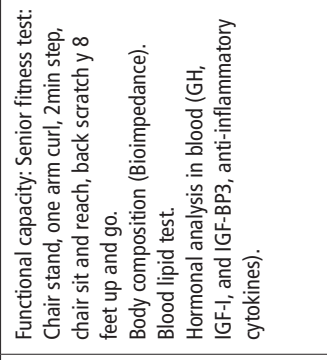 & 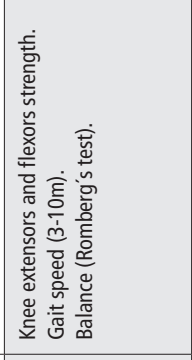 & 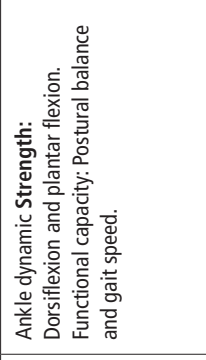 \\
\hline 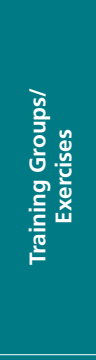 & 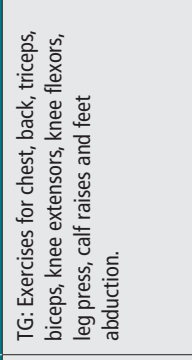 & 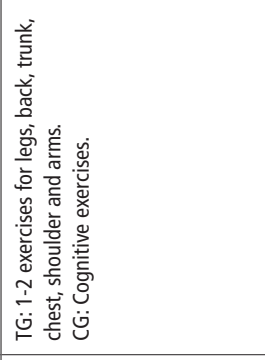 & 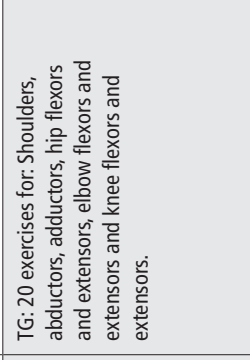 & 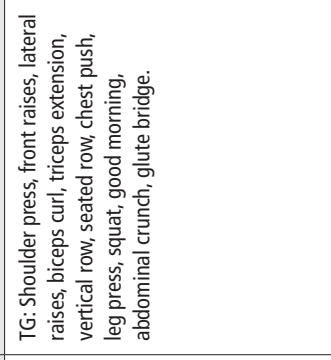 & 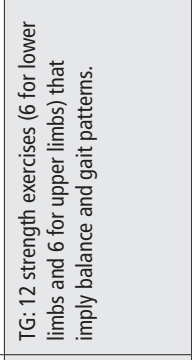 & 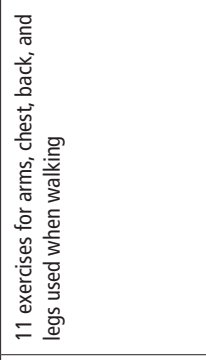 \\
\hline 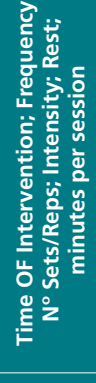 & 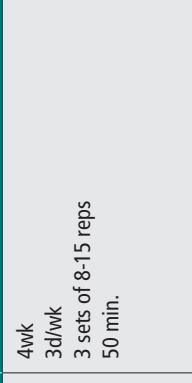 & 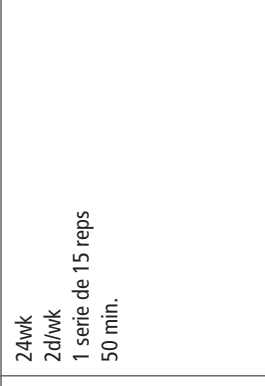 & 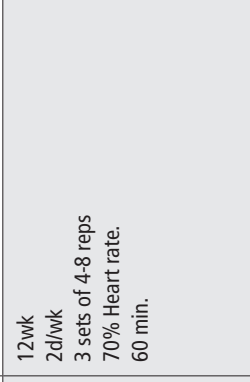 & 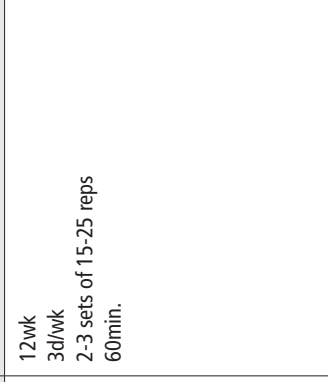 & 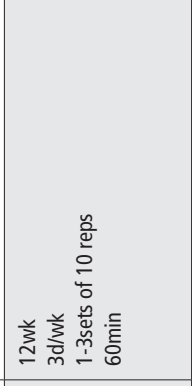 & 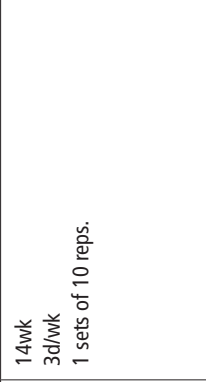 \\
\hline 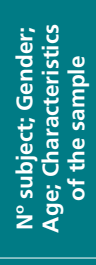 & 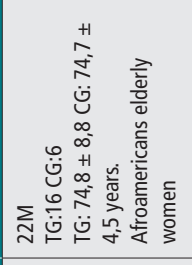 & 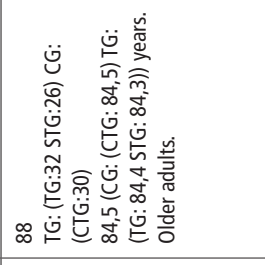 & 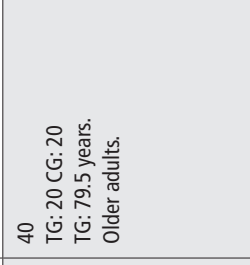 & 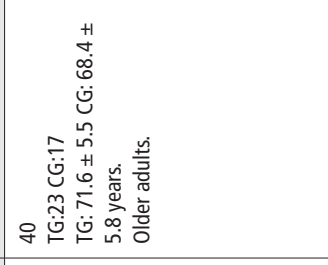 & 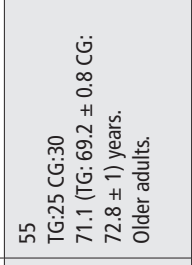 & 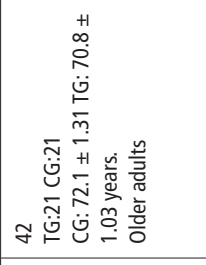 \\
\hline 总 & 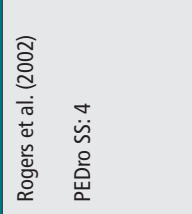 & 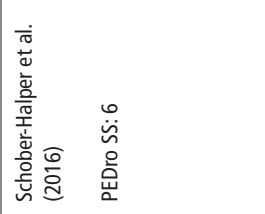 & 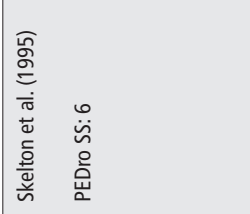 & 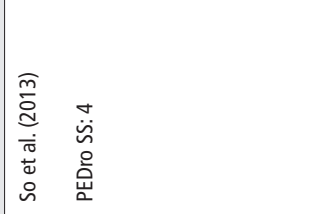 & 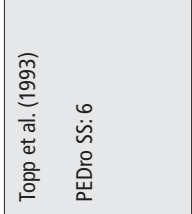 & 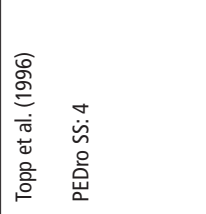 \\
\hline
\end{tabular}




\begin{tabular}{|c|c|c|c|c|c|}
\hline$\frac{2}{5}$ & 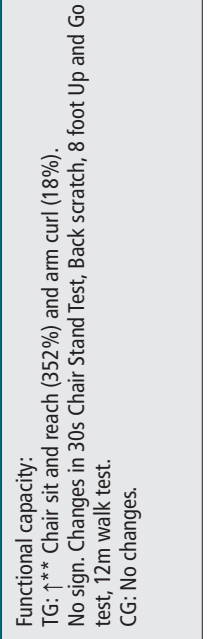 & 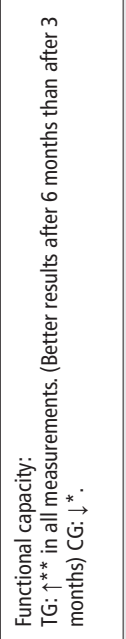 & 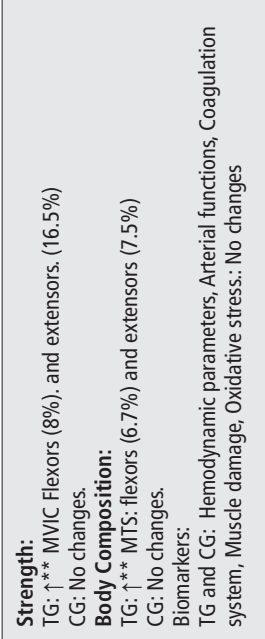 & 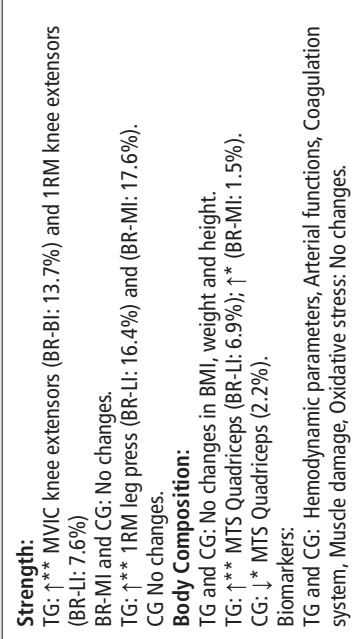 & 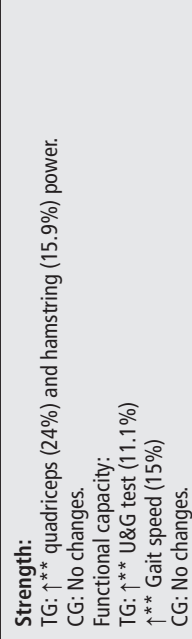 \\
\hline 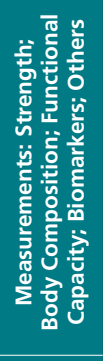 & 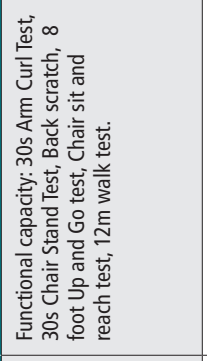 & 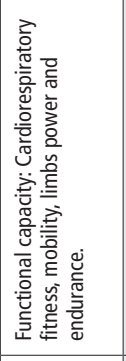 & 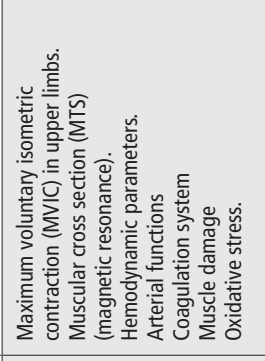 & 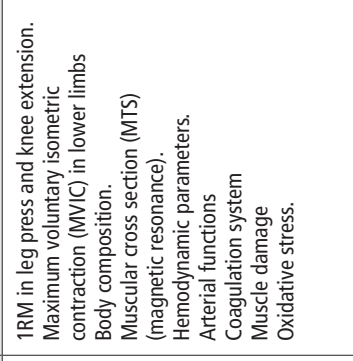 & 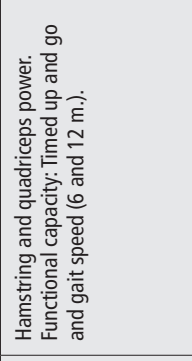 \\
\hline 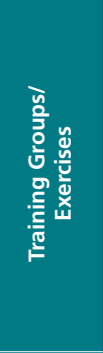 & 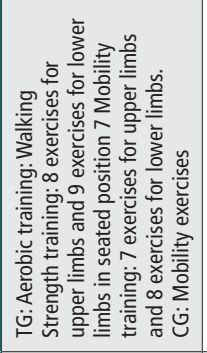 & 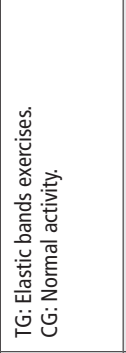 & 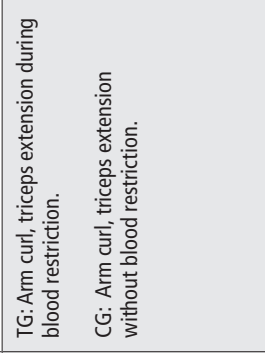 & 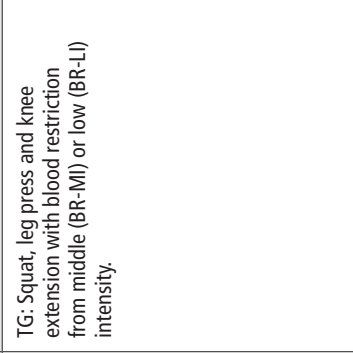 & 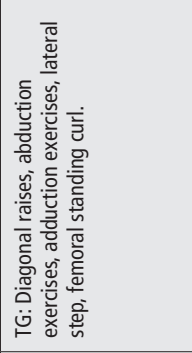 \\
\hline 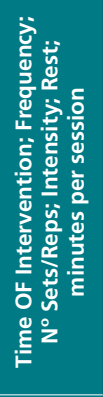 & 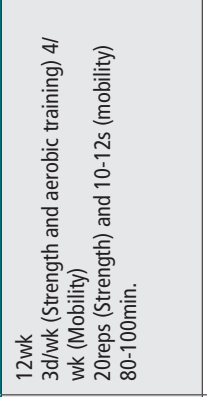 & 姜窇言 & 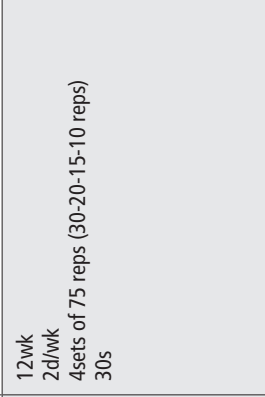 & 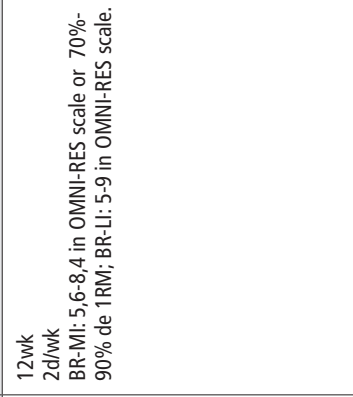 & 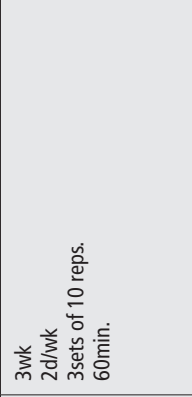 \\
\hline 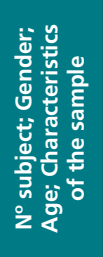 & 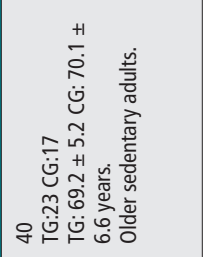 & 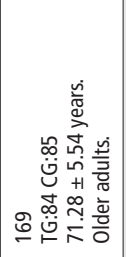 & 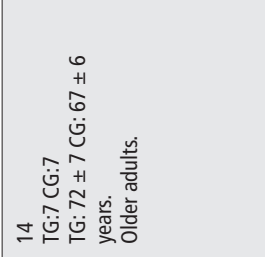 & 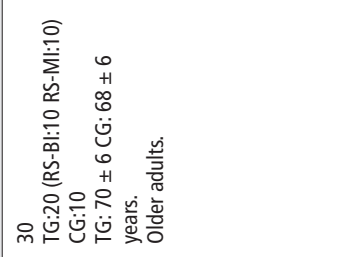 & 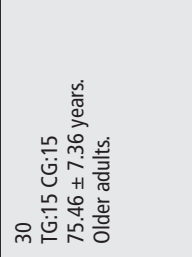 \\
\hline 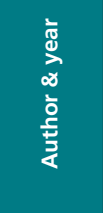 & 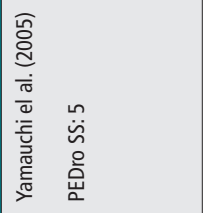 & 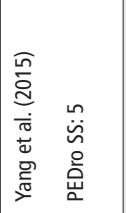 & 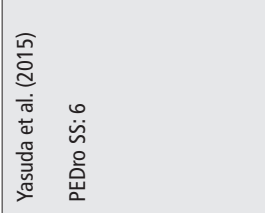 & 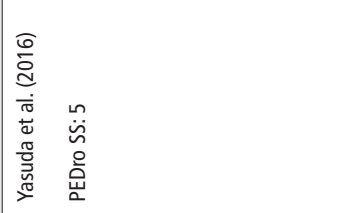 & 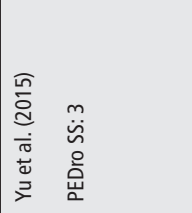 \\
\hline
\end{tabular}

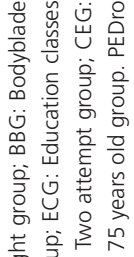

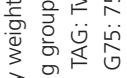

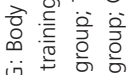

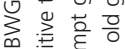

言它焉峞

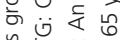

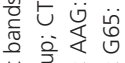

套亭 言商

要

总密

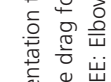

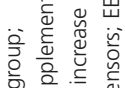

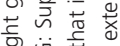

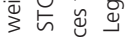

这穹害获

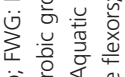

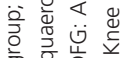

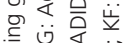

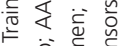

过 言 产离

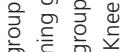

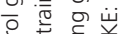

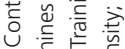

نे

突它它

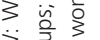

응 을

这. 을 흥

is

管

पू

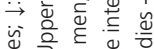

\% 응

놀 흫

$\leftarrow$ 을 흥

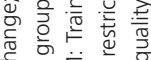

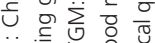

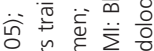

年

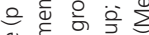

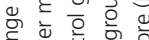

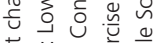

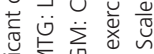

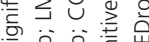

䍬言言 
Aboodarda, S. J., Page, P. A., \& Behm, D. G. (2016). Muscle activation comparisons between elastic and isoinertial resistance: A metaanalysis. Clinical Biomechanics, 39, 52-61. doi:10.1016/j.clinbiomech. 2016.09.008

Aboodarda, S. J., Hamid, M. S. A., Che Muhamed, A. M., Ibrahim, F., \& Thompson, M. (2013). Resultant muscle torque and electromyographic activity during high intensity elastic resistance and free weight exercises. European Journal of Sport Science, 13(2), 155-163. doi:10.10 80/17461391.2011.586438

Aniansson, A., Ljungberg, P., Rundgren, Å., \& Wetterqvist, H. (1984). Effect of a training programme for pensioners on condition and muscular strength. Archives of Gerontology and Geriatrics, 3(3), 229-241. doi:10.1016/0167-4943(84)90024-4

Annesi, J., Westcott, W., Faigenbaum, A., Unruh, J. (2005). Effect of a 12 week physical activity program deliverede by YMCA after-school conselors (Youth fit for life) on fitness and self-efficacy changes in 5-12 year old boys and girls. Research Quarterly for Exercise and Sport, 76 468-476.

Barkley, J. E., Ryan, E. J., Bellar, D., Bliss, M. V., \& Roemmich, J. N. (2011). The variety of exercise equipment and physical activity participation in children. Journal of Sport Behavior, 34(2), 137.

Behm, D. G. (1991). An Analysis of Intermediate Speed Resistance Exercises for Velocity-specific Strength Gains. The Journal of Strength \& Conditioning Research, 5(1), 1-5.doi:10.1519/00124278-19910200000001

Bellar, D. M., Muller, M. D., Barkley, J. E., Kim, C. H., Ida, K., Ryan, E. J., Bliss, M. V., \& Glickman, E. L. (2011). The effects of combined elastic-and free-weight tension vs. free-weight tension on one-repetition maximum strength in the bench press. The Journal of Strength \& Conditioning Research, 25(2), 459-463. doi:10.1519/JSC.0b013e3181c1f8b6

Braith, R. W., \& Stewart, K. J. (2006). Resistance exercise training. Circulation, 113(22), 2642-2650. doi:10.1161/CIRCULATIONAHA. 105.584060

Calatayud, J., Borreani, S., Colado, J. C., Martin, F., Tella, V., \& Andersen, L. L. (2015). Bench press and push-up at comparable levels of muscle activity results in similar strength gains. The Journal of Strength \& Conditioning Research, 29(1), 246-253. doi:10.1519/ JSC.0000000000000589

Capodaglio, P., Edda, M. C., Facioli, M., \& Saibene, F. (2007). Long-term strength training for community-dwelling people over 75: impact on muscle function, functional ability and life style. European journal of applied physiology, 100(5), 535-542. doi:10.1007/s00421-006-0195-8

Capodaglio, P., Facioli, M., Burroni, E., Giordano, A., Ferri, A., \& Scaglioni, G. (2002). Effectiveness of a home-based strengthening program for elderly males in Italy. A preliminary study. Aging clinical and experimental research, 14(1), 28-34. doi:10.1007/BF03324414

Capodaglio, P., Ferri, A., \& Scaglioni, G. (2005). Effects of a partially supervised training program in subjects over 75 years of age. Aging clinical and experimental research, 17(3), 174-180. doi:10.1007/ BF03324593

Chupel, M. U., Direito, F., Furtado, G. E., Minuzzi, L. G., Pedrosa, F. M., Colado, J. C., Ferreira, J. P., Filaire, E., \& Teixeira, A. M. (2017). Strength Training Decreases Inflammation and Increases Cognition and Physical Fitness in Older Women with Cognitive Impairment. Frontiers in physiology, 8, 377. doi:10.3389/fphys.2017.00377.

Clark, B. C., \& Manini, T. M. (2008). Sarcopenia $\neq$ dynapenia. The Journals of Gerontology Series A: Biological Sciences and Medical Sciences, 63(8), 829-834. doi:10.1093/gerona/63.8.829

Colado, J. C., García-Massó, X., Pellicer Catalán, M., Alakhdar, Y., Benavent, J., \& Cabeza Ruiz, R. (2010). A comparison of elastic tubing and isotonic resistance exercises. International journal of sports medicine, 31(11), 810817. doi:10.1055/s-0030-1262808

Colado, J. C, Garcia-Masso, X., Rogers, M., Tella, V., Benavent, J., \& Dantas, E. (2012a). Effects of aquatic and dry land resistance training devices on body composition and physical capacity in postmenopausal women. Journal of human kinetics, 32, 185-195. doi:10.2478/v10078 012-0035-3
Colado, J. C., \& Triplett, N. T. (2008). Effects of a short-term resistance program using elastic bands versus weight machines for sedentary middle-aged women. The Journal of Strength \& Conditioning Research, 22(5), 1441-1448. doi:10.1519/JSC.0b013e31817ae67a

Colado, J. C., Garcia-Masso, X., Triplett, T. N., Flandez, J., Borreani, S., \& Tella, V. (2012b). Concurrent validation of the OMNI-resistance exercise scale of perceived exertion with Thera-band resistance bands. The Journal of Strength \& Conditioning Research, 26(11), 30183024. doi:10.1519/JSC.0b013e318245c0c9

Colado, J. C., Triplett, N. T., Tella, V., Saucedo, P., \& Abellán, J. (2009). Effects of aquatic resistance training on health and fitness in postmenopausal women. European journal of applied physiology, 106(1), 113-122. doi:10.2478/v10078-012-0035-3

Coskun, A., \& Sahin, G. (2014). Two different strength training and untrained period effects in children. Journal of Physical Education and Sport, 14(1), 42. doi:10.7752/jpes.2014.01007

Cyarto, E. V., Brown, W. J., Marshall, A. L., \& Trost, S. G. (2008) Comparison of the effects of a home-based and group-based resistance training program on functional ability in older adults. American Journal of Health Promotion, 23(1), 13-17. doi:10.4278/ajhp.07030120

Damush, T. M., \& Damush, J. G. (1999). The effects of strength training on strength and health-related quality of life in older adult women. The Gerontologist, 39(6), 705-710. doi:10.1093/geront/39.6.705

de Oliveira, P. A., Blasczyk, J. C., Junior, G. S., Lagoa, K. F., Soares, M., de Oliveira, R. J., et al. (2017). Effects of elastic resistance exercise on muscle strength and functional performance in healthy adults: a systematic review and meta-analysis. Journal of physical activity and health, 14, 317-327. doi:10.1123/jpah.2016-0415

Escala PEDro. (2017). Recuperado el 11 de Mayo de 2017 de: https://www. pedro.org.au/spanish/downloads/pedro-statistics/.

Faigenbaum, A. D. (2000). Strength training for children and adolescents. Clinics in sports medicine, 19(4), 593-619. doi:10.1016/ S0278-5919(05)70228-3

Flandez, J., Belando, N., Gargallo, P., Fernández-Garrido, J., VargasFoitzick, R. A., Devis-Devis, J., \& Colado, J. C. (2017). Metabolic and functional profile of premenopausal women with metabolic syndrome after training with elastics as compared to free weights. Biological research for nursing, 19(2), 190-197. doi:10.1177/1099800416674307

Franzke, B., Halper, B., Hofmann, M., Oesen, S., Jandrasits, W., Baierl, A., et al. (2015a). The impact of six months strength training, nutritional supplementation or cognitive training on DNA damage in institutionalised elderly. Mutagenesis, 30(1), 147-153. doi:10.1093/ mutage/geu074

Franzke, B., Halper, B., Hofmann, M., Oesen, S., Pierson, B., Cremer, A., et al. (2015b). The effect of six months of elastic band resistance training, nutritional supplementation or cognitive training on chromosomal damage in institutionalized elderly. Experimental gerontology, 65, 16-22. doi:10.1016/j.exger.2015.03.001

Furtado; G., Carvalho, H. M., Loureiro, M., Patrício, M., Uba-Chupel, M., Colado, J. C., Hogervorst, E., Ferreira, J. P., \& Ana Maria Teixeira, A. M. (2019). Chair-based Exercise Programs in Institutionalized Older Women: Salivary Steroid Hormones, Disabilities and Frailty Changes. Experimental Gerontology, 1107902019 Dec 6[Online ahead of print] doi:10.1016/j.exger.2019.110790

Gargallo, P., Colado, J. C., Juesas, A., Hernando-Espinilla, A., EstañCapell, N., Monzó-Beltran, L., García-Pérez, P., Cauli, O., \& Sáez, G. T. (2018). The Effect of Moderate- Versus High-Intensity Resistance Training on Systemic Redox State and DNA Damage in Healthy Older Women. Biological research for nursing, 20(2), 205-217. doi:10.1177/1099800417753877

Gómez-Álvarez, N., Jofré-Hermosilla, N., Matus-Castillo, C., \& Pavez-Adasme, G. (2019). Effects of muscle strength training in postmenopausal women with metabolic syndrome. Systematic review. Cultura_Ciencia_Deporte, 14(42), 213-224.

Granacher, U., Lesinski, M., Büsch, D., Muehlbauer, T., Prieske, O., Puta, C., Gollhofer, A., \& Behm, D. G. (2016). Effects of Resistance Training in Youth Athletes on Muscular Fitness and Athletic Performance: A 
Conceptual Model for Long-Term Athlete Development. Frontiers in physiology, 7, 164. doi:10.3389/fphys.2016.00164

Harnirattisai, T., Thongtawee, B., \& Raetong, P. (2015). The Effects of a Physical Activity Program for Fall Prevention among Thai Older Adults. Pacific Rim International Journal of Nursing Research, 19(1), 4-18. doi:10.1111/ggi.13052

Heislein, D. M., Harris, B. A., \& Jette, A. M. (1994). A strength training program for postmenopausal women: a pilot study. Archives of physical Medicine and Rehabilitation, 75(2), 198-204

Hofmann, M., Schober-Halper, B., Oesen, S., Franzke, B., Tschan, H., Bachl, N., et al. (2016). Effects of elastic band resistance training and nutritional supplementation on muscle quality and circulating muscle growth and degradation factors of institutionalized elderly women: the Vienna Active Ageing Study (VAAS). European journal of applied physiology, 116(5), 885-897. doi:10.1007/s00421-016-3344-8

Hostler, D. C., Schwirian, I., Campos, G., Toma, K., Crill, M. T., Hagerman, G. R., Hagerman, F. C., \& Staron, R. S. (2001). Skeletal muscle adaptations in elastic resistance-trained young men and women. European journal of applied physiology, 86(2), 112-118. doi:10.1007/ s004210100495

Hughes, C. J., \& McBride, A. (2005). The use of surface electromyography to determine muscle activation during isotonic and elastic resistance exercises for shoulder rehabilitation. Orthopaedic physical therapy practice, $17(2), 18$.

Ignjatović, A., Stanković, R., Radovanović, D., Marković, Ž., \& Cvećka, J. (2009). Resistance training for youths. Facta Universitatis: Series Physical Education and Sport, 7(2), 189-196.

Iwamoto, J. (2013). Effects of physical activity on bone: what type of physical activity and how much is optimal for bone health. Journal of Osteoporis and Physical Activity, 1, 101.doi:10.4172/23299509.1000e101

Jakobsen, M. D., Sundstrup, E., Andersen, C. H., Aagaard, P., \& Andersen, L. L. (2013). Muscle activity during leg strengthening exercise using free weights and elastic resistance: effects of ballistic vs controlled contractions. Human Movement Science, 32(1), 65-78. doi:10.1016/j. humov.2012.07.002

Jonghwan, C., Chulhyun, R., Kyumoon, L., Hyeonju, K., \& Byeungok, L. (2003). Effect of the PNF and weight training on flexibility, muscular strength, and power in college males. Journal of Physical Growth and Motor Development, 11, 35-43.

Kompf, J., \& Arandjelović, O. (2016). Understanding and overcoming the sticking point in resistance exercise. Sports Medicine, 46(6), 751-762. doi:10.1007/s40279-015-0460-2

Kwak, C. J., Kim, Y. L., \& Lee, S. M. (2016). Effects of elastic-band resistance exercise on balance, mobility and gait function, flexibility and fall efficacy in elderly people. Journal of physical therapy science, 28(11), 3189-3196. doi:10.1589/jpts.28.3189

Lee, H. C., Lee, M. L., \& Kim, S. R. (2015). Effect of exercise performance by elderly women on balance ability and muscle function. Journal of physical therapy science, 27(4), 989-992. doi:10.1589/jpts.27.989

Liao, L. Y., Chung, W. S., \& Chen, K. M. (2017). Free radicals and antioxidant enzymes in older adults after regular senior elastic band exercising: an experimental randomized controlled pilot study. Journal of Advanced Nursing, 73(1), 108-111. doi:10.1111/jan.13094

Lubans, D. R., Aguiar, E. J., \& Callister, R. (2010). The effects of free weights and elastic tubing resistance training on physical selfperception in adolescents. Psychology of Sport and Exercise, 11(6), 497504. doi:10.1016/j.ypmed.2009.12.003

Lubans, D. R., Mundey, C. M., Lubans, N. J., \& Lonsdale, C. C. (2013). Pilot randomized controlled trial: elastic-resistance-training and lifestyleactivity intervention for sedentary older adults. Journal of aging and physical activity, 21(1), 20-32. doi:10.1123/japa.21.1.20

Maher, C. G., Sherrington, C., Herbert, R. D., Moseley, A. M., \& Elkins, M. (2003). Reliability of the PEDro scale for rating quality of randomized controlled trials. Physical therapy, 83(8), 713. doi:10.1093/ptj/83.8.713

Martins, W. R., de Oliveira, R. J., Carvalho, R. S., de Oliveira Damasceno, V., da Silva, V. Z. M., \& Silva, M. S. (2013). Elastic resistance training to increase muscle strength in elderly: a systematic review with metaanalysis. Archives of gerontology and geriatrics, 57(1), 8-15. doi:10.1016/j. archger.2013.03.002
Martins, W. R., Safons, M. P., Bottaro, M., Blasczyk, J. C., Diniz, L. R., Fonseca, R. M. C., et al. (2015). Effects of short term elastic resistance training on muscle mass and strength in untrained older adults: a randomized clinical trial. BMC geriatrics, 15(1), 99. doi:10.1186/ s12877-015-0101-5

Matheson, J. W., Kernozek, T. W., Fater, D. C., \& Davies, G. J. (2001). Electromyographic activity and applied load during seated quadriceps exercises. Medicine and science in sports and exercise, 33(10), 1713-1725. doi:10.1097/00005768-200110000-00016

Mikesky, A. E., Topp, R., Wigglesworth, J. K., Harsha, D. M., \& Edwards, J. E. (1994). Efficacy of a home-based training program for older adults using elastic tubing. European journal of applied physiology and occupational physiology, 69(4), 316-320. doi:10.1007/BF00392037

Moher, D., Liberati, A., Tetzlaff, J., Altman, D. G., \& Prisma Group. (2009). Preferred reporting items for systematic reviews and meta-analyses: the PRISMA statement. PLoS medicine, 6(7), e1000097. doi:10.1186/20464053-4-1

Oesen, S., Halper, B., Hofmann, M., Jandrasits, W., Franzke, B., Strasser, E. M., et al. (2015). Effects of elastic band resistance training and nutritional supplementation on physical performance of institutionalised elderly: a randomized controlled trial. Experimental gerontology, 72, 99-108. doi:10.1016/j.exger.2015.08.013

Oh, S. L., Kim, H. J., Woo, S., Cho, B. L., Song, M., Park, Y. H., et al. (2016). Effects of an integrated health education and elastic band resistance training program on physical function and muscle strength in community-dwelling elderly women: Healthy Aging and Happy Aging II study. Geriatrics \& gerontology international, 17(5), 825-833. doi:10.1111/ggi.12795

Page, P., \& Ellenbecker, T. S. (2003). The scientific and clinical application of elastic resistance. Champaign, IL: Human Kinetics.

Papadopoulos, C., \& Jager, J. M. (2016). The effect of an educational program on strength-training adherence in older adults. Educational Gerontology, 42(5), 342-351. doi:10.1093/fampra/cmu069

Park, B. S., Khamoui, A. V., Brown, L. E., Kim, D. Y., Han, K. A., Min, K. W., \& An, G. H. (2016). Effects of elastic band resistance training on glucose control, body composition, and physical function in women with shortvs. long-duration type-2 diabetes. The Journal of Strength \& Conditioning Research, 30(6), 1688-1699. doi:10.1519/JSC.0000000000001256

Park, S. Y., Kim, J. K., \& Lee, S. A. (2015). The effects of a communitycentered muscle strengthening exercise program using an elastic band on the physical abilities and quality of life of the rural elderly. Journal of physical therapy science, 27(7), 2061-2063. doi:10.1589/jpts.27.2061

Rhyu, H. S., Kim, S. H., \& Park, H. S. (2015). The effects of band exercise using proprioceptive neuromuscular facilitation on muscular strength in lower extremity. Journal of exercise rehabilitation, 11(1), 36. doi:10.12965/jer.150189

Rieping, T., Furtado, G. E., Letieri, R. V., Chupel, M. U., Colado, J. C., Hogervorst, E., Filaire, E., Teixeira, A. M. M. B., \& Ferreira, J. P. (2019). Effects of Different Chair-Based Exercises on Salivary Biomarkers and Functional Autonomy in Institutionalized Older Women. Research quarterly for exercise and sport, 90(1), 36-45. doi:10.1080/02701367.2 018.1563272

Rogers, M. E., Sherwood, H. S., Rogers, N. L., \& Bohlken, R. M. (2002). Effects of dumbbell and elastic band training on physical function in older inner-city African-American women. Women \& health, 36(4), 3341. doi: 10.1300/J013v36n04_03

Rosenberg, I. H. (1997). Sarcopenia: origins and clinical relevance. The Journal of nutrition, 127(5), 990S-991S. doi:10.1093/jn/127.5.990S

Sahin, G., Aslan, M., \& Demir, E. (2016). Short-term effect of back squat with an elastic band on the squat and vertical jump performance in trained children. Journal of Physical Education and Sport, 16(1), 97. doi:10.7752/jpes.2016.01016

Schober-Halper, B., Hofmann, M., Oesen, S., Franzke, B., Wolf, T., Strasser, E. M., et al. (2016). Elastic band resistance training influences transforming growth factor-ß receptor I mRNA expression in peripheral mononuclear cells of institutionalised older adults: the Vienna Active Ageing Study (VAAS). Immunity \& Ageing, 13(1), 22. doi:10.1186/ s12979-016-0077-9

Shin, S. O., \& Kim, N. S. (2016). Accessory Respiratory Muscle Activation during Chest Expansion Exercise using Elastic Bands in Children with 
Cerebral Palsy. Korean Society of Physical Medicine, 11(3), 119-124. doi:10.13066/kspm.2016.11.3.119

Skelton, D. A., Young, A., Greig, C. A., \& Malbut, K. E. (1995). Effects of resistance training on strength, power, and selected functional abilities of women aged 75 and older. Journal of the American Geriatrics Society, 43(10), 1081-1087. doi:10.1111/j.1532-5415.1995.tb07004.x

So, W. Y., Song, M., Park, Y. H., Cho, B. L., Lim, J. Y., Kim, S. H., \& Song, W. (2013). Body composition, fitness level, anabolic hormones, and inflammatory cytokines in the elderly: a randomized controlled trial. Aging clinical and experimental research, 25(2), 167-174. doi:10.1007/s40520-013-0032-y

Soria-Gila, M. A., Chirosa, I. J., Bautista, I. J., Baena, S., \& Chirosa, L. J. (2015). Effects of variable resistance training on maximal strength: a meta-Analysis. The Journal of Strength \& Conditioning Research, 29(11), 3260-3270. doi:10.1519/JSC.0000000000000971

Suchomel, T. J., Nimphius, S., Bellon, C. R., \& Stone, M. H. (2018). The importance of muscular strength: Training considerations. Sports Medicine, 48(4),765-785. doi:10.1007/s40279-018-0862-z

Sugimoto, D., \& Blanpied, P. (2006). Flexible foil exercise and shoulder internal and external rotation strength. Journal of athletic training, 41(3), 280

Thiebaud, R. S., Loenneke, J. P., Fahs, C. A., Rossow, L. M., Kim, D., Abe, T., et al. (2013). The effects of elastic band resistance training combined with blood flow restriction on strength, total bone-free lean body mass and muscle thickness in postmenopausal women. Clinical physiology and functional imaging, 33(5), 344-352. doi:10.1111/cpf.12033

Thorborg, K., Bandholm, T., Petersen, J., Weeke, K. M., Weinold, C., Andersen, B., et al. (2010). Hip abduction strength training in the clinical setting: with or without external loading? Scandinavian journal of medicine \& science in sports, 20(s2), 70-77. doi:10.1111/j.16000838.2010.01186.x

Thorborg, K., Bandholm, T., Zebis, M., Andersen, L. L., Jensen, J., \& Hölmich, P. (2016). Large strengthening effect of a hip-flexor training programme: a randomized controlled trial. Knee Surgery, Sports Traumatology, Arthroscopy, 24(7), 2346-2352. doi:10.1007/s00167 015-3583-y

Topp, R., Mikesky, A., Dayhoff, N. E., \& Holt, W. (1996). Effect of resistance training on strength, postural control, and gait velocity among older adults. Clinical Nursing Research, 5(4), 407-427. doi:10. 1177/105477389600500404

Topp, R., Mikesky, A., Wigglesworth, J., Holt, W., \& Edwards, J. E. (1993). The effect of a 12-week dynamic resistance strength training program on gait velocity and balance of older adults. The Gerontologist, 33(4), 501-506. doi:10.1093/geront/33.4.501

Vincent, K. R., Vincent, H. K., Braith, R. W., Bhatnagar, V., \& Lowenthal, D. T. (2003). Strength training and hemodynamic responses to exercise. The American journal of geriatric cardiology, 12(2), 97-106. doi:10.1111/j.1076-7460.2003.01588.x

Winters-Stone, K. M., \& Snow, C. M. (2006). Site-specific response of bone to exercise in premenopausal women. Bone, 39(6), 1203-1209. doi:10.1016/j.bone.2006.06.005

Yamauchi, T., Islam, M. M., Koizumi, D., Rogers, M. E., Rogers, N. L., \& Takeshima, N. (2005). Effect of home-based well-rounded exercise in community-dwelling older adults. Journal of Sports Science and Medicine, 4(4), 563-571.

Yang, H. J., Chen, K. M., Chen, M. D., Wu, H. C., Chang, W. J., Wang, Y C., \& Huang, H. T. (2015). Applying the transtheoretical model to promote functional fitness of community older adults participating in elastic band exercises. Journal of advanced nursing, 71(10), 2338-2349. doi:10.1111/jan.12705

Yasuda, T., Fukumura, K., Iida, H., \& Nakajima, T. (2015). Effects of detraining after blood flow-restricted low-load elastic band training on muscle size and arterial stiffness in older women. Springerplus, 15(4), 348. doi:10.1186/s40064-015-1132-2.

Yasuda, T., Fukumura, K., Fukuda, T., Uchida, Y., Iida, H., Meguro, M., et al. (2014). Muscle size and arterial stiffness after blood flow-restricted low-intensity resistance training in older adults. Scandinavian journal of medicine \& science in sports, 24(5), 799-806. doi:10.1111/sms.12087

Yasuda, T., Fukumura, K., Tomaru, T., \& Nakajima, T. (2016). Thigh muscle size and vascular function after blood flow-restricted elastic band training in older women. Oncotarget, 7(23), 33595. doi:10.18632/ oncotarget.9564

Yu, S., Lee, Y., \& Kim, S. (2015). Effect of Elastic-Band Exercise and Cognitive Rehabilitation in Cognition and Walking Speed of Elderly People-Pilot Study. Journal of the Ergonomics Society of Korea, 34(5), 363-375. doi:10.5143/JESK.2015.34.5.363 